ARTICLE

Received 30 Oct 2014 | Accepted 8 Jun 2015 | Published 24 Jul 2015

DOI: $10.1038 /$ ncomms 8743 OPEN

\title{
MEG3 long noncoding RNA regulates the TGF- $\beta$ pathway genes through formation of RNA-DNA triplex structures
}

\author{
Tanmoy Mondal', Santhilal Subhash ${ }^{1}$, Roshan Vaid ${ }^{1}$, Stefan Enroth², Sireesha Uday ${ }^{1}$, Björn Reinius ${ }^{1}$, \\ Sanhita Mitra1, Arif Mohammed', Alva Rani James', Emily Hoberg ${ }^{3}$, Aristidis Moustakas ${ }^{4,5}$, \\ Ulf Gyllensten ${ }^{2}$, Steven J.M. Jones ${ }^{6}$, Claes M. Gustafsson ${ }^{3}$, Andrew H. Sims ${ }^{7}$, Fredrik Westerlund ${ }^{8}$, \\ Eduardo Gorab ${ }^{9} \&$ Chandrasekhar Kanduri ${ }^{1}$
}

Long noncoding RNAs (IncRNAs) regulate gene expression by association with chromatin, but how they target chromatin remains poorly understood. We have used chromatin RNA immunoprecipitation-coupled high-throughput sequencing to identify 276 IncRNAs enriched in repressive chromatin from breast cancer cells. Using one of the chromatin-interacting IncRNAs, MEG3, we explore the mechanisms by which IncRNAs target chromatin. Here we show that MEG3 and EZH2 share common target genes, including the TGF- $\beta$ pathway genes. Genome-wide mapping of MEG3 binding sites reveals that MEG3 modulates the activity of TGF- $\beta$ genes by binding to distal regulatory elements. MEG3 binding sites have GA-rich sequences, which guide MEG3 to the chromatin through RNA-DNA triplex formation. We have found that RNA-DNA triplex structures are widespread and are present over the MEG3 binding sites associated with the TGF- $\beta$ pathway genes. Our findings suggest that RNA-DNA triplex formation could be a general characteristic of target gene recognition by the chromatin-interacting IncRNAs.

\footnotetext{
${ }^{1}$ Department of Medical Genetics, Institute of Biomedicine, The Sahlgrenska Academy, University of Gothenburg, SE-40530 Gothenburg, Sweden.

${ }^{2}$ Department of Immunology, Genetics and Pathology, Biomedical Center, SciLifeLab Uppsala, Uppsala University, SE-75108 Uppsala, Sweden. ${ }^{3}$ Department of Medical Biochemistry and Cell Biology, University of Gothenburg, PO Box 440, SE-405 30 Gothenburg, Sweden. ${ }^{4}$ Department of Medical Biochemistry and Microbiology, Science for Life Laboratory, Uppsala University, PO Box 582, SE-751 23 Uppsala, Sweden. ${ }^{5}$ Ludwig Institute for Cancer Research, Science for Life Laboratory, Uppsala University, PO Box 595, SE-751 24 Uppsala, Sweden. ${ }^{6}$ Genome Sciences Centre, British Columbia Cancer Agency, Vancouver, British Columbia BC V5Z 4S6, Canada. 7 Applied Bioinformatics of Cancer, University of Edinburgh Cancer Research UK Centre, Edinburgh EH4 2 XR, UK. ${ }^{8}$ Department of Chemical and Biological Engineering, Chalmers University of Technology, Gothenburg 412 96, Sweden. ${ }^{9}$ Departamento de Genética e Biologia Evolutiva, Instituto de Biociências, Universidade de São Paulo, São Paulo CEP:05508-090, Brazil. Correspondence and requests for materials should be addressed to C.K. (email: Kanduri.chandrasekhar@gu.se).
} 
ong noncoding RNAs (lncRNAs) have emerged as key regulators of important biological processes implicated in development and differentiation ${ }^{1-6}$. Studies on the mode of action of lncRNAs have revealed that a subset of $\operatorname{lncRNAs}$ regulate gene expression in cis and trans by interacting with chromatin and recruiting chromatin modifiers ${ }^{7-12}$. Most studies to date have focused on identification of the RNA-interacting protein partners involved in gene activation or gene silencing ${ }^{13-16}$, and less attention has been paid in understanding how lncRNAs specifically target genes. Nevertheless, some recent investigations have provided insights into Xist targeting and its spreading along the inactive $\mathrm{X}$ chromosome $(\mathrm{Xi})^{17}{ }^{18}$. These studies did not predict any consensus binding sites by which Xist RNA is initially recruited before spreading along the $\mathrm{Xi}$, but it has been proposed that the three-dimensional chromosomal conformation may play an important role in Xist spreading. On the other hand, chromatin-binding maps of HOTAIR and Drosophila roX2 lncRNAs revealed that GA-rich sequences are the preferred binding motif, indicating that GA-rich sequences may help these RNAs to target the chromatin ${ }^{19}$. Identification of the lncRNAs that are associated with chromatin and exploration of the mechanistic aspects of the chromatin targeting of lncRNAs will help us to understand the molecular intricacies underlying lncRNA-dependent gene expression at the transcriptional level.

Active and inactive epigenetic modifications of the chromatin can regulate gene expression at the transcriptional level. When chromatin is enriched with repressive histone marks such as $\mathrm{H} 3 \mathrm{~K} 27 \mathrm{me} 3$ and $\mathrm{H} 3 \mathrm{~K} 9 \mathrm{me} 3$, it negatively regulates transcription ${ }^{20}$. The H3K27me3 histone modification is mediated by polycomb repressive complex 2 (PRC2). EZH2, EED and SUZ12 are the three major components of the PRC2 complex, where EZH2 is the catalytic subunit and EED is known to help in the propagation of $\mathrm{H} 3 \mathrm{~K} 27 \mathrm{me} 3$ marks by allosteric activation of PRC2 (refs 21,22). In Drosophila, the recruitment of PRC2 to the chromatin is mediated by specific sequences known as polycomb response elements. However, in mammals, it is not clear how sequence-specific recruitment of the PRC2 occurs across the genome. Recent evidence of a strong association between lncRNA and the PRC2 complex raise the possibility that lncRNAs may act as guiding molecules for PRC2 to target the chromatin ${ }^{23}$.

Several previous studies have focused on the identification of the polycomb-interacting lncRNAs either by using the RNA immunoprecipitation (RIP) technique or the photoactivatable ribonucleside-enhanced crosslinking and immunoprecipitation technique ${ }^{24-27}$. Although these studies have identified several
PRC2-interacting lncRNAs, it remains unclear whether these lncRNAs are targeted to the chromatin.

Hence we sought to identify the repressive chromatinassociated lncRNAs on a global scale and also characterize the mechanisms by which these lncRNAs are targeted to the chromatin. Here we have characterized the repressive chromatinassociated lncRNAs on a genome-wide scale by performing chromatin RIP followed by high-throughput sequencing (ChRIP-seq) using antibodies to H3K27me3 and EZH2 in BT-549 cells. We identified 276 lncRNAs that are enriched in repressive chromatin. By using one of the chromatin-interacting lncRNAs (MEG3) as a model system, we explored the mechanisms by which it recognizes target genes. Consistent with ChRIP-seq data, MEG3 interacts with the PRC2 complex. Through loss-of-function experiments of MEG3 and EZH2, we found that MEG3 in cooperation with PRC2 regulates a common set of genes, including those of the transforming growth factor- $\beta$ (TGF- $\beta$ ) pathway. Using a modified chromatin oligo affinity precipitation (ChOP) method, we fine-mapped genome-wide chromatin-binding sites for MEG3 RNA, revealing some of the TGF- $\beta$ pathway genes as direct targets. MEG3 binding sites showed enrichment in GA-rich sequences and we found that these GA-rich sequences guide MEG3 RNA to its target genes through formation of RNA-DNA triplex structures. Our data demonstrate that RNA-DNA triplex structures are widespread in vivo, and are also present in the vicinity of the TGF- $\beta$ pathway genes. Taken together, these results suggest that RNA-DNA triplex formation may be a general mechanism for target gene recognition by lncRNAs.

\section{Results}

Characterization of repressive chromatin-enriched lncRNAs. Previously, we have used ChRIP to verify the chromatin association of the mouse Kcnqlot1 antisense lncRNA ${ }^{10}$. Here we used a modified ChRIP protocol in combination with photoactivatable ribonucleside-enhanced crosslinking followed by high-throughput sequencing (ChRIP-seq) to identify lncRNAs that are associated with repressive chromatin on a global scale (Fig. 1a). In brief, we incubated BT-549 cells overnight (14-16 h) with 4-thiouridine (4sU), followed by a 40-min incubation with actinomycin D (ActD). ActD-treated BT-549 cells were crosslinked with formaldehyde, followed by ultraviolet irradiation. 4sU-incorporated RNA can be crosslinked with proteins in vivo by ultraviolet irradiation. Crosslinking with formaldehyde ensures stabilization of the chromatin-interacting

\footnotetext{
Figure 1 | Identification of repressive chromatin-associated IncRNAs using ChRIP-seq. (a) The ChRIP-seq analysis pipeline used to identify IncRNAs enriched in repressive chromatin. The pie chart shows 276 IncRNAs enriched in both EZH2 and H3K27me3 ChRIP-seq samples compared with the nuclear RNA (input). The $P$ value was obtained by performing a hypergeometric test using all the IncRNAs in our analysis. (b) Bar diagram showing the distribution of T-to-C transitions (indicative of putative RNA-protein contact sites) in input $(8,361)$, EZH2 $(18,905)$ and H3K27me3 $(2,651)$ ChRIP-seq data. Black in the EZH2 bar indicates the number of T-to-C transitions $(1,253)$ that are either present in input or H3K27me3 samples, and blue indicates EZH2-specific T-to-C transitions $(17,652)$. The EZH2-specific T-to-C transitions $(17,652)$ were used to associate with IncRNAs. (c) All the possible conversions present in the EZH2 ChRIP-seq sample. T-to-C conversion and the reverse-strand A-to-G conversions were predominant among all the possible conversion events. (d) LncRNAs (1,046; annotated and non-annotated) harbour EZH2-specific $(17,652)$ T-to-C conversion site. Seventy repressive chromatin-enriched IncRNAs (out of 276) carry T-to-C transitions, including known PRC2-interacting IncRNAs such as MEG3, KCNQ1OT1 and BDNF-AS1. The P value was obtained by performing a hypergeometric test using all the IncRNAs considered in our analysis. (e,f) The distribution of the sequencing reads on MEG3 and KCNQ1OT1 transcripts from H3K27me3, EZH2-enriched chromatin fractions and input RNA samples. The tags represent the read distribution and the signal represents the intensity of reads over MEG3 and KCNQ10T1 transcripts. Locations of T-to-C transitions over the exons are depicted below the physical maps. The left panel depicts the RPKM (Reads per kilobase per million) for MEG3 and KCNQ1OT1 in H3K27me3, EZH2 ChRIP RNA and input RNA samples. The fold enrichment (FC) in H3K27me3 and EZH2 ChRIP RNA compared with input is indicated. (g) ChRIP validation: RT-qPCR data showing the enrichment of the selected annotated and non-annotated IncRNAs in the EZH2 and H3K27me3 ChRIP pull-downs compared with input. We did not observe any enrichment of these IncRNAs in the H3K4me2 (active chromatin marks) and immunoglobulin G (IgG; nonspecific antibody) ChRIP pull-downs. Actin was used as a negative control. Data represent the mean \pm s.d. of three independent biological experiments.
} 
lncRNAs to the chromatin. Incubation of BT-549 cells with ActD before crosslinking blocks transcription, which in turn prevents the co-transcriptional crosslinking of lncRNAs to the chromatin. The efficacy of the transcriptional arrest by ActD was tested using short half-life mRNA $C-M Y C$ as described previously (Supplementary Fig. 1a) ${ }^{28}$. Chromatin was prepared from the formaldehyde and ultraviolet -crosslinked BT-549 cells, and was subjected to immunoprecipitation using antibodies to H3K27me3 a

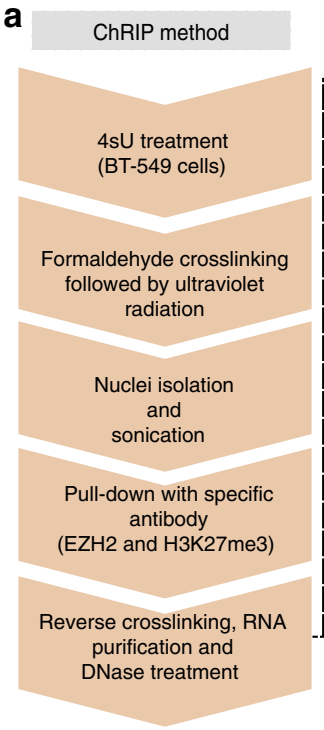

b

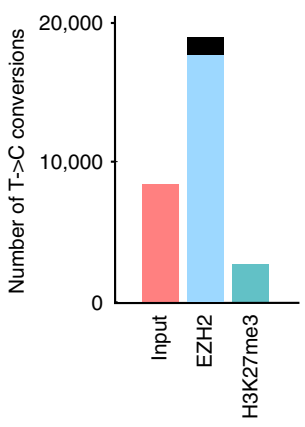

Computational analysis

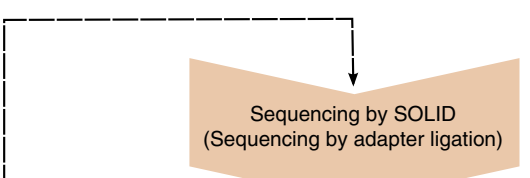

(Sequencing by adapter ligation)

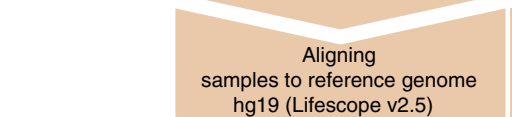

hg19 (Lifescope v2.5)

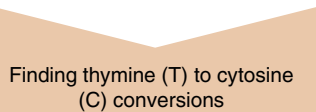

(C) conversions

(Freebayes v0.6.3)

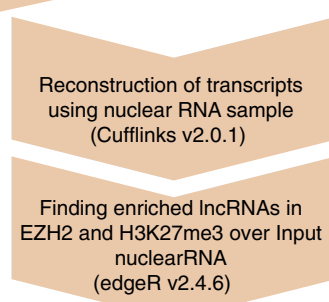

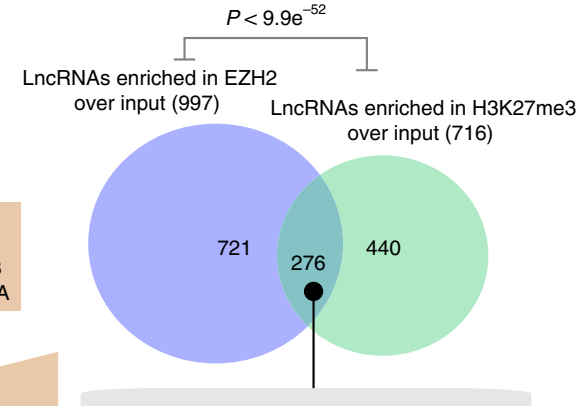

Repressive chromatin enriched IncRNAs
C

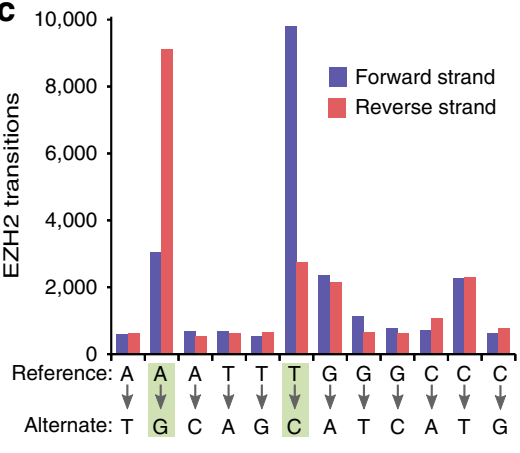

d

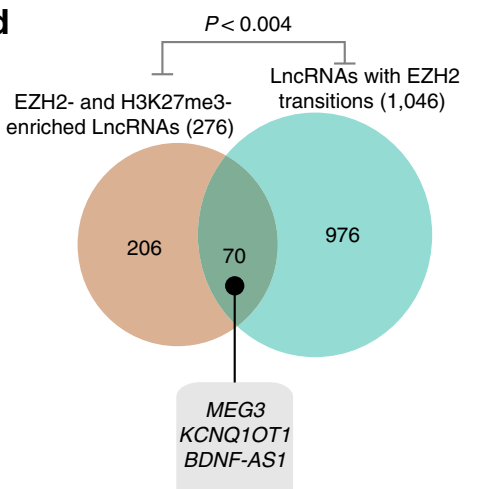

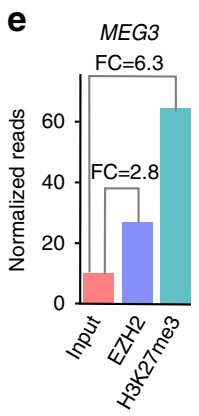

RefSeq genes
EZH2 trans
EZH2 tags
EZH2 signal
H3K27me3 tags
H3K27me3 signal
Input tags
Input signal

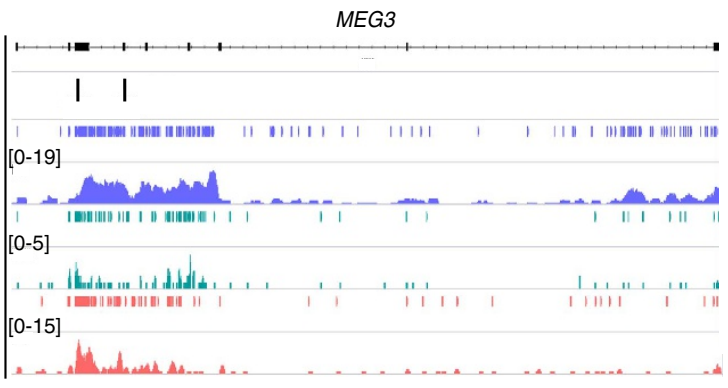

f

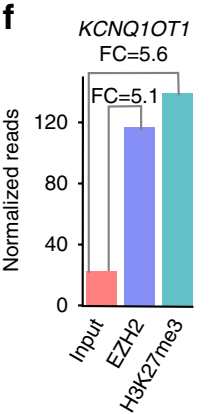

KCNQ1OT1

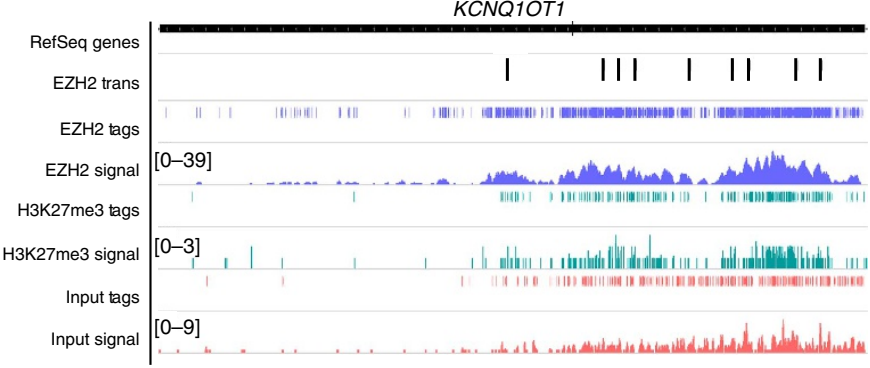

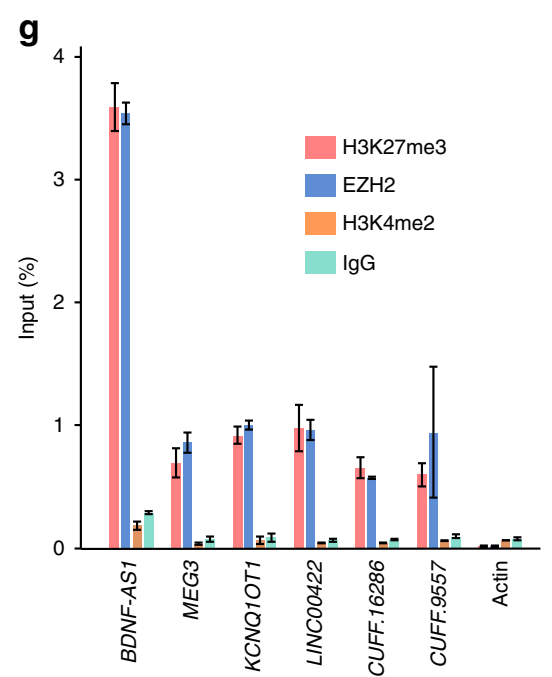


and EZH2. The specificity of the immunopurified chromatin was tested by quantitative PCR (qPCR) with positive and negative controls (Supplementary Fig. 1b). After reversal of crosslinking, RNA was isolated from the immunoprecipitated chromatin. Isolated RNA was extensively treated with DNase I to remove all traces of DNA, and verified again by qPCR (Supplementary Fig. 1b). The DNase I-treated anti-H3K27me3 and anti-EZH2 purified RNAs along with nuclear input RNA were subjected to high-throughput sequencing. The reconstruction of the nuclear RNA using Cufflinks revealed previously annotated lncRNAs and also non-annotated transcripts. Coding potential analysis of the non-annotated transcripts found that they had lower coding probabilities, suggesting that they are noncoding RNAs (Supplementary Fig. 1c). We looked for enrichment of the annotated and non-annotated transcripts in $\mathrm{H} 3 \mathrm{~K} 27 \mathrm{me} 3$ and EZH2 ChRIP-purified RNA fractions over nuclear input (Supplementary Data 1 and 2). We considered lncRNAs in our ChRIP data set to be 'repressive chromatin enriched' only if they were enriched (minimum twofold) in both H3K27me3 and EZH2 ChRIP-purified RNA fractions compared with the nuclear input. We found a significant overlap (276 lncRNAs, $P<9.9 \mathrm{e}^{-52}$, hypergeometric distribution) between H3K27me3 and EZH2 ChRIP pull-downs (Fig. 1a). The list of 276 lncRNAs enriched in repressive chromatin comprises both annotated and non-annotated transcripts (Supplementary Data 3). The $4 \mathrm{sU}$ incorporation provided us with an additional advantage in our RNA sequencing data, as the possible protein interaction sites on RNA lead to ultraviolet-induced T-to-C transitions. The T-to-C conversions at the putative RNA-protein contact sites in ChRIP RNA sequencing samples were considered only if the minimum sequencing read depth over the conversions was $\geq 2$ (read depth indicates total number of sequencing reads covered per transition $)^{29}$. Using this criterion, we observed that T-to-C conversion was overrepresented in the EZH2 ChRIP RNA fraction in comparison with both the H3K27me3 ChRIP RNA and input RNA (Fig. 1b). We found that the overrepresentation of T-to-C conversion in EZH2 ChRIP data was not by chance, as the other nucleotide conversions were detected at background level in the EZH2 ChRIP data compared with T to C (A to G also represents T-to-C conversion in the reverse strand of RNA sequencing data; Fig. 1c). We identified 17,652 T-to-C conversions that were present only in EZH2 ChRIP data but not in H3K27me3 and input RNA data. These T-to-C conversions were then mapped to annotated and non-annotated transcripts, reconstructed from nuclear RNA input, revealing 1,046 lncRNAs with putative RNA-protein contact sites. We found a significant overlap between these $\operatorname{lncRNAs}$ and the $\operatorname{lncRNAs}$ that were enriched in EZH2 ChRIP and in repressive chromatin (enriched in both H3K27me3 and EZH2 ChRIPs) (Fig. 1d, Supplementary Fig. 1d and Supplementary Data 4). The presence of EZH2 ChRIP-specific T-to-C conversion sites over the repressive chromatin-associated IncRNAs indicates that they are either putative EZH2 contact sites or EZH2-associated protein contact sites over the lncRNAs. Interestingly, the 70 repressive chromatin-associated lncRNAs with T-to-C conversions contain several annotated and non-annotated (both intergenic and intronic) lncRNAs (Supplementary Data 4 and Supplementary Fig. 2a,b), including three known PRC2-interacting lncRNAs: KCNQ1OT1, MEG3 and BDNF-AS1 (Fig. 1e,f and Supplementary Data 4). Mouse orthologues Kcnq1ot1 and Gtl2 have been shown to interact with PRC2, and moreover Kcnq1ot1 has also been shown to be enriched in the mouse placental chromatin fraction $^{10,24,30}$. We validated the repressive chromatin enrichment of some of the annotated lncRNAs (BDNF-AS1, MEG3, KCNQ1OT1 and LINC00422) and non-annotated lncRNAs (intergenic CUFF.16286 and intronic CUFF.9557) using $\mathrm{qPCR}$ assay on ActD-treated and -untreated ChRIP materials (Fig. 1g and Supplementary Fig. 2c).

Mapping PRC2-interacting region of MEG3 lncRNA. Since MEG3 lncRNA was identified as a repressive chromatin-associated RNA in the ChRIP analysis (Fig. 1d,e), and also as one of the chromatin-interacting RNAs in our previous study involving sucrose-fractionated chromatin from normal human fibroblasts (HF cells) ${ }^{28}$, we were interested in exploring plausible mechanisms by which MEG3 lncRNA recognizes its target genes. Human MEG3 is an lncRNA of $\sim 1,700$ nucleotides with different isoforms generated by alternative splicing. Exons $1-3$ and 8-9 are common to all isoforms, whereas exons 4-7 are present in different combinations ${ }^{31}$. In situ RNA hybridization and nuclearcytoplasmic RNA fractionation experiments indicated that MEG3 is located in the nuclear compartment (Fig. 2a,b). We checked for the interaction of MEG3 lncRNA with PRC2 by RIP and found robust enrichment of MEG3 in the PRC2-interacting RNA fraction (Fig. 2c), and its fold enrichment was more or less similar to the enrichment of KCNQ1OT1 lncRNA (Fig. 2c), which was used as positive control for the RIP experiment.

We then wanted to fine-map the sequences of $M E G 3$ RNA that dictate interaction of PRC2 with MEG3. To this end, we looked for the status of the incorporated $4 \mathrm{sU}$ nucleotide conversions ( $\mathrm{T}$ to $\mathrm{C}$ ) in the EZH2 ChRIP-seq data, which indicates possible RNA-protein contact points. We detected two converted nucleotides at the $5^{\prime}$-end of the MEG3 RNA in the EZH2

Figure 2 | Molecular characterization of MEG3 and PRC2 interaction. (a) RNA-fluorescence in situ hybridization showing the distribution of the MEG3 signal (green) in the nucleus (blue, stained with 4,6-diamidino-2-phenylindole). An RNase A-treated sample was used as a negative control. Scale bar, $1 \mu \mathrm{m}$. (b) RT-qPCR data showing the distribution of IncRNAs and protein-coding RNAs in the nuclear and cytoplasmic fractions ( \pm s.d., $n=3$ ). (c) RT-qPCR analysis of MEG3, KCNQ1OT1 and U1SnRNA in EZH2 RIP-purified RNA from BT-549 cells. U1SnRNA served as negative control. The enrichment is plotted as percentage of input ( \pm s.d., $n=3$ ). (d) Physical map of the MEG3 containing numbered exons showing two T-to- $C$ transitions. The exons in red are constitutively expressed and blue are alternatively spliced exons. First conversion is part of exon 3 showing higher expression, whereas the second conversion is part of exon 4 showing low expression in the nuclear RNA sequencing. (e) In vitro interaction of MEG3 and PRC2. The schematic indicates the exons of the WT MEG3 clone. Left: RT-qPCR showing enrichment of sense WT MEG3 and MEG3 carrying deletions ( $\triangle 340-348$ or $\triangle 345-348$ MEG3) in in vitro RNA binding assays. Reaction with antisense WT MEG3 or without purified PRC2 served as negative controls. The binding efficiency of MEG3 deletions were presented relative to WT MEG3 ( \pm s.d., $n=3$ ). Right: RT-qPCR showing the quantification of input RNAs. (f) Upper panel: western blot showing EZH2 levels after pull-down with biotinylated sense WT MEG3, antisense WT MEG3, and $\triangle 345-348$ MEG3 RNAs incubated with nuclear extract. This is a representative data set from several experiments. Lower panel: agarose gel picture showing input biotin-RNA. (g) RT-qPCR result showing the relative enrichment of WT MEG3, $3340-348$ and $\triangle 345-348$ MEG3 RNAs in the EZH2-RIP, performed after BT-549 cells were transfected with WT and mutant MEG3 plasmids. Data were normalized to the input RNAs and plotted as percentage of input ( \pm s.d., $n=3$ ). To distinguish the endogenous $M E G 3$ from the ectopically expressed MEG3, we designed RT-qPCRs primers, with one primer mapped to the transcribed portion of the vector and the other to MEG3 RNA. Endogenous MEG3 served as positive control and U1SnRNA as negative control. 
immunopurified RNA (Figs 1e and 2d), indicating that the conversions could be possible contact points for EZH2 or EZH2associated protein contact sites. The first conversion was located in a constitutively expressed exon 3 , whereas the second one was in an alternatively spliced exon 4 (ref. 31). We PCR-amplified full-length MEG3 clones using nuclear RNA from BT-549 cells (hereon referred to as wild-type (WT) MEG3). It contains all the constitutively expressed exons (that is, exons 1-3 and 8-9) and an alternatively spliced exon 7 (out of the four alternatively spliced exons 4-7), but not exon 4. Moreover, we detected fewer reads in the nuclear RNA over the alternatively spliced exon with the second T-to-C conversion (Fig. 2d). Since we failed to detect the
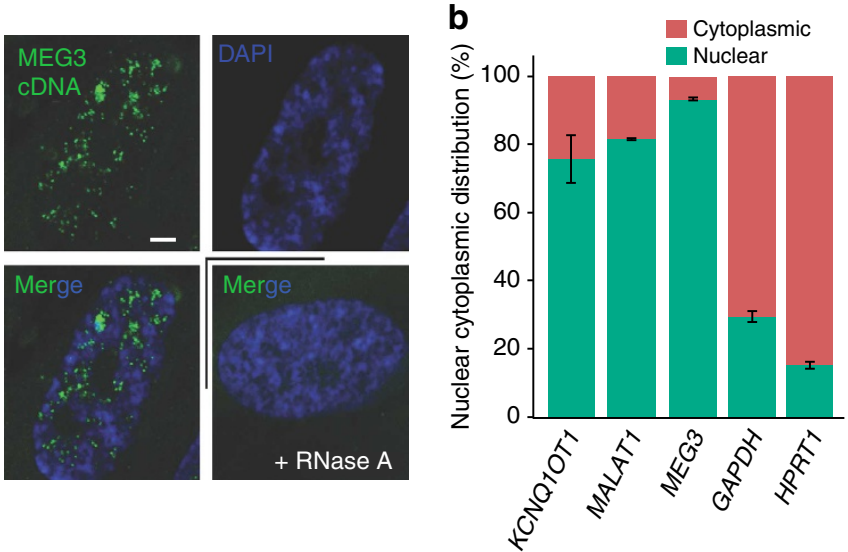

C

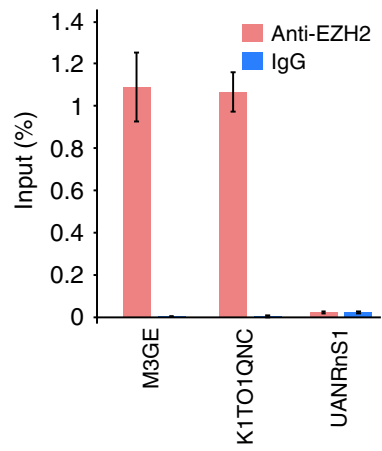

d
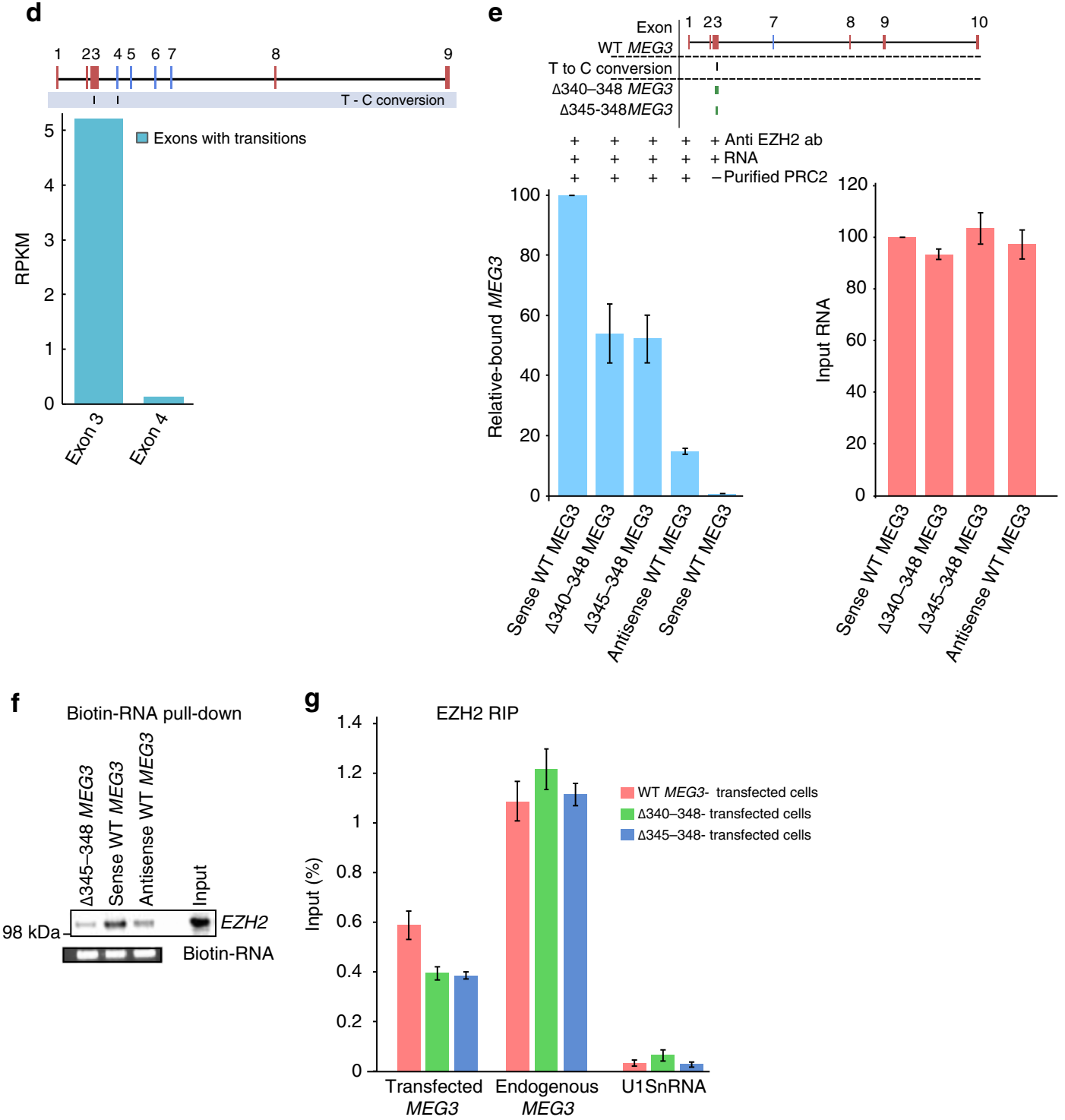
alternatively spliced exon 4 in several full-length amplified MEG3 clones, we therefore focused on the first T-to-C conversion located in the constitutively expressed exon 3. We inserted two deletions of $4 \mathrm{bp}(345-348)$ and $9 \mathrm{bp}$ (340-348) (Fig. 2e), overlapping the first conversion site in exon 3 , into a WT $M E G 3$ clone. We carried out an in vitro binding assay with WT and mutant ( $\triangle 340-348$ MEG3 and $\triangle 345-348$ MEG3) MEG3 RNAs using purified PRC2 complex, comprising EZH2, EED and SUZ12 subunits (Fig. 2e and Supplementary Fig. 3a). An antisense version of the full-length MEG3 (antisense WT MEG3) was used as a negative control. We found that the PRC2 binding was partially compromised in the mutant MEG3 RNAs ( $\triangle 340-348$ MEG3 and $\triangle 345-348$ MEG3) compared with the WT MEG3 (Fig. 2e). We also tested the binding of WT and $\triangle 345-348$ MEG3 RNAs with PRC2 using the nuclear lysate from BT-549 cells, and found that the sense WT MEG3, but not the $\triangle 345-348$ MEG3 RNA, efficiently bound to PRC2, as detected by EZH2 immunoblot (Fig. 2f). To further test the efficacy of the association of PRC2 with the WT and mutant MEG3 RNAs in vivo, we transfected BT-549 cells with the plasmids expressing WT, $\Delta 340-348$ and $\Delta 345-348$ MEG3 RNAs, and performed RIP. By using primer combinations that selectively amplify the transfected plasmid-derived MEG3 RNAs, we found that $\triangle 340-348$ MEG3 and $\triangle 345-348$ MEG3 transcripts were less enriched than the WT-transfected MEG3, whereas endogenous MEG3 was equally enriched in all pull-downs (Fig. 2g). Thus, by using $4 \mathrm{~s} \mathrm{U}$ conversion data along with in vitro and in vivo binding experiments, we had fine-mapped the probable contact points for PRC2 interaction in the human MEG3 lncRNA.

MEG3/EZH2 interaction regulates the TGF- $\beta$ pathway genes. To gain more insights into the functional significance of the interaction of PRC2 with the MEG3 lncRNA, both EZH2 and MEG3 transcripts were downregulated in BT-549 and HF cells using small interfering RNA (siRNA), and gene expression profiles were measured using microarray. We observed a significant overlap among the deregulated genes between the EZH2 and MEG3 data sets from BT-549 and HF cells, indicating a functional association between the MEG3 lncRNA and EZH2 (Fig. 3a, Supplementary Data 5 and Supplementary Data 6). MEG3 downregulation did not interfere with $E Z H 2$ RNA and protein levels, and similarly, EZH2 downregulation did not affect $M E G 3$ transcript levels (Fig. 3b,c), suggesting that the overlap observed between the deregulated genes in the EZH2 and MEG3 data sets was not due to changes in EZH2 levels upon MEG3 downregulation or in $M E G 3$ levels upon $E Z H 2$ downregulation. To rule out off-target effects of the MEG3 siRNA, we used reverse transcription (RT)-qPCR to validate four target genes upon $M E G 3$ downregulation using an alternative siRNA against MEG3 (Supplementary Fig. 3b,c).

Pathway analysis of the differentially expressed genes identified from microarray revealed that several pathways were affected in common after MEG3 and EZH2 removal (Table 1 and Supplementary Data 7). We also performed RNA sequencing of the $M E G 3 / E Z H 2$ downregulated samples from an independent biological experiment, and found a significant overlap among differentially expressed genes from the RNA sequencing and microarray experiments (Supplementary Fig. 4a). Pathway analysis of the RNA sequencing samples also revealed the same pathways as those obtained using microarray experiments, further suggesting a functional interaction between MEG3 and EZH2 (Table 1 and Supplementary Data 7). In addition, similar pathways were obtained with the commonly deregulated genes from microarray and RNA sequencing experiments upon MEG3 and EZH2 downregulation (Supplementary Fig. 4b). Since the
TGF- $\beta$ pathway is one of the well-investigated signalling cascades in mammals among the affected pathways, we were interested in understanding the functional role of $M E G 3$ in the regulation of genes involved in the TGF- $\beta$ pathway. We validated the differential expression of the key TGF- $\beta$ pathway genes TGFB2, TGFBR1 and SMAD2 in BT-549 and HF cells after siRNAmediated downregulation of EZH2 and $M E G 3$ transcripts (Fig. 3d,e and Supplementary Fig. 4c). The overexpression of full-length $M E G 3$ and $E Z H 2$ resulted in significant downregulation of the TGF- $\beta$ pathway genes TGFB2, TGFBR1 and SMAD2 (Fig. $3 \mathrm{f}$ and Supplementary Fig. 4d). Activation of the TGF- $\beta$ pathway target genes ACTC1, CNN1 and COL5A1, with functional roles in cytoskeletal organization, was also observed upon downregulation of EZH2 and $M E G 3$ by siRNA (Supplementary Fig. 5a). We further confirmed the TGF- $\beta$ pathway-mediated regulation of these genes by treating BT-549 cells with TGF- $\beta 2$ ligand for $24 \mathrm{~h}$, and, as expected, we observed activation of expression of the ACTC1, CNN1 and COL5A1 genes (Supplementary Fig. 5b). We also observed an additive effect on the activation of these genes if the cells were treated with both MEG3 siRNA and TGF- $\beta$ ligand together (Supplementary Fig. 5c). Activation of these genes was not observed when the cells were treated with MEG3 siRNA along with a TGF- $\beta$ inhibitor (Supplementary Fig. 5c). These results together highlight how $M E G 3$ regulates its secondary gene targets through control of the primary TGF- $\beta$ target genes.

Considering the functional role of the TGF- $\beta$ pathway in the regulation of cell invasion ${ }^{32-34}$, we investigated whether the activated the TGF- $\beta$ pathway members in the MEG3downregulated cells enhance cell invasion. To this end, we performed a Matrigel cell invasion assay with the BT-549 cells transduced with lentiviral particles containing MEG3 and control short hairpin RNAs (shRNAs). Compared with the control shRNA, the MEG3 shRNA-transduced BT-549 cells showed activation of the TGF- $\beta$ pathway genes (Supplementary Fig. $5 \mathrm{~d}$ ) and also showed a significant increase in their invasion through Matrigel (Fig. 3g). The increased invasion of the MEG3 shRNA cells was reversed when the cells were treated with TGF- $\beta$ inhibitor (Fig. 3g). We also observed an increase in the cell invasion of the control shRNA-expressing cells in the presence of TGF- $\beta 2$ ligand (Fig. $3 g$ ). These results suggest that MEG3 partly controls the cell invasion of BT-549 cells through regulation of the TGF- $\beta$ pathway. When we overexpressed MEG3 in MDAMB-231 cells, we found that there was a significant decrease in the invasive capacity of the MDA-MB-231 cells, indicating that MEG3 RNA suppresses cell invasion (Supplementary Fig. 5e).

Given the functional interaction between $M E G 3$ expression and TGF- $\beta$ gene regulation in the breast cancer cell line BT-549, we extended our cell line analyses to the published clinical breast cancer data sets. We found that MEG3 is expressed at significantly lower levels in invasive ductal carcinoma than in normal breast tissue (Supplementary Fig. 5f). We then integrated gene expression data from 17 published studies, representing 2,999 primary breast tumours, and found that MEG3 had the lowest average expression and widest range of expression in the aggressive and difficult-totreat basal molecular subtype (Supplementary Fig. 5g). Consistent with this observation, MEG3 is expressed at lower levels in highgrade breast tumours (Supplementary Fig. 5h). We also observed that TGFB2, TGFBR1 and SMAD2 genes (Supplementary Fig. 5i) had greater expression in tumours with low MEG3 expression, further supporting our in vitro cell culture results that MEG3 negatively regulates the TGF- $\beta$ pathway genes (Fig. 3).

MEG3 binds to distal regulatory elements of TGF- $\beta$ genes. We next wanted to address an important question: how does MEG3 
a
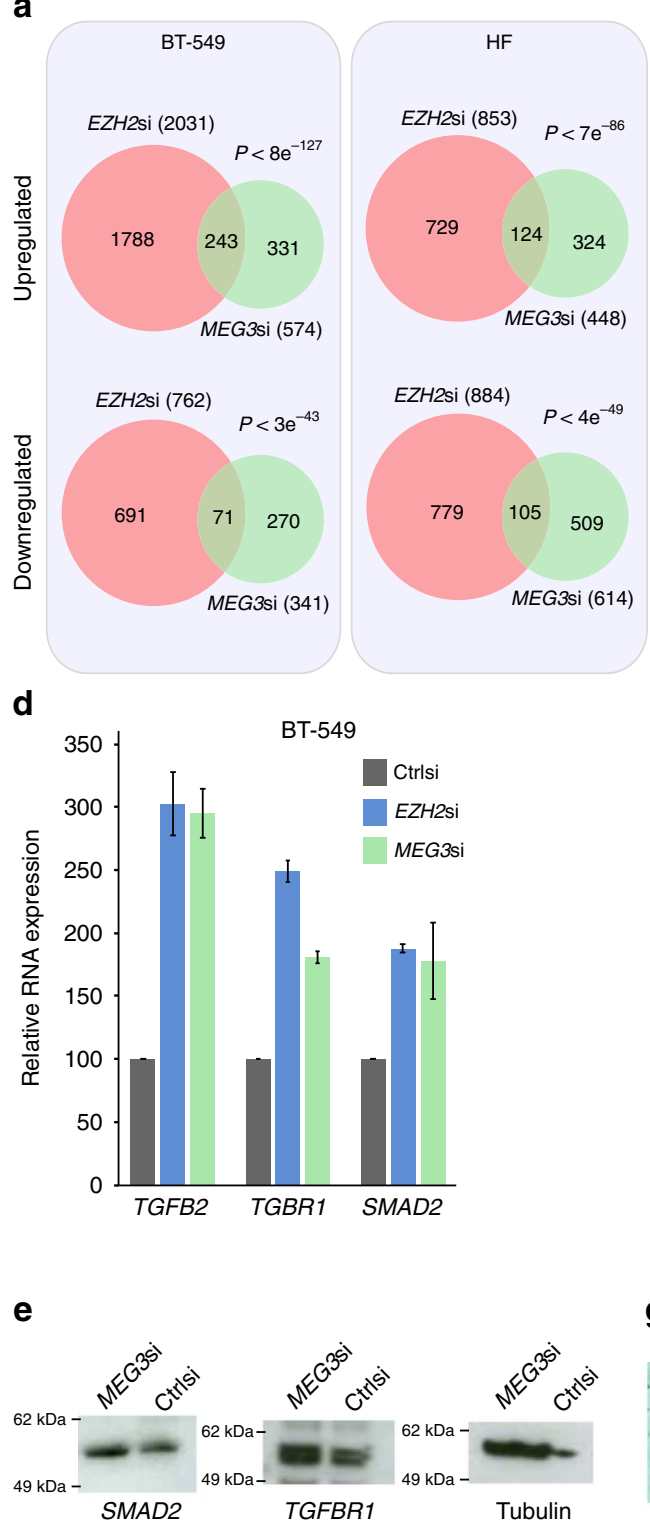

b
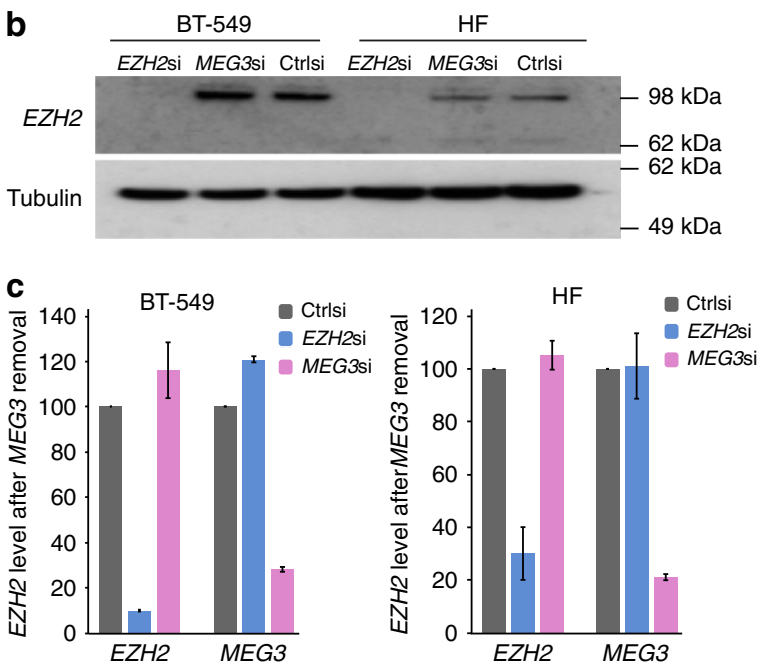

f
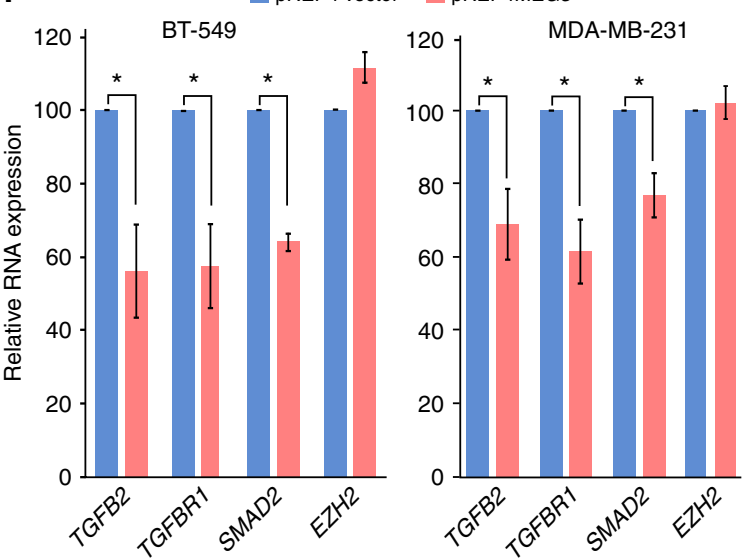

g

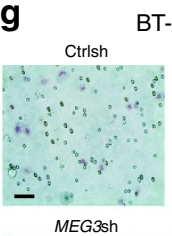

BT-549

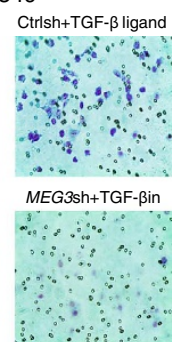

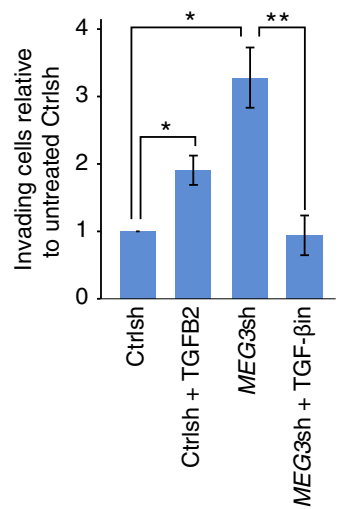

Figure 3 | MEG3/EZH2 functional interaction regulates TGF- $\beta$ pathway genes. (a-c) MEG3 and EZH2 share common gene targets. (a) Venn diagram showing the number of genes deregulated after downregulation of MEG3 and EZH2 using siRNA in BT-549 and HF cells, and the degree of overlap between the MEG3- and EZH2-dependent genes. The $P$ values were obtained by hypergeometric test using all protein-coding genes as a background. (b) EZH2 protein levels, as determined by western blotting, following EZH2 and MEG3 downregulation in BT-549 and HF cells. Tubulin was used as a loading control. (c) RT-qPCR analysis of EZH2 and MEG3 mRNA expression in Ctrlsi, EZH2si and MEG3si transfected BT-549 and HF cells ( \pm s.d., $n=3$ ). (d) RT-qPCR analysis of TGFB2, TGFBR1 and SMAD2 gene expression in Ctrlsi, MEG3si and EZH2si transfected BT-549 cells $( \pm$ s.d., $n=3$ ). (e) Immunoblot showing SMAD2, TGFBR1 and tubulin protein levels following transfection of BT-549 cells with Ctrlsi and MEG3si. (f) Bar graph showing RT-qPCR analysis of TGFB2, TGFBR1 and SMAD2 mRNA levels after overexpression of MEG3 (pREP4MEG3) in BT-549 and MDA-MB-231 cells. The levels in pREP4MEG3 are presented relative to CtrlpREP4 ( \pm s.d., $n=3$ ). EZH2 was used as a control showing no change in expression after overexpression of $M E G 3$. The $P$ values were calculated using Student's $t$-test (two-tailed, two-sample unequal variance), ${ }^{\star} P<0.05$. (g) Downregulation of MEG3 influences the invasive property of BT-549 cells through regulation of the TGF- $\beta$ pathway. Images showing Matrigel invasion of the BT-549 cells. The two images in the upper panel show invasion of BT-549 cells infected with Ctrlsh lentivirus, and Ctrlsh infection followed by incubation with TGF- $\beta 2$ ligand (Ctrlsh-TGFB2). The images in the bottom panel show the cells infected with MEG3Sh and MEG3sh infection followed by incubation with TGF- $\beta$ inhibitor (MEG3sh + TGF- $\beta$ in). Scale bar, 10 $\mu \mathrm{m}$. The bar graph shows quantification ( $\pm \mathrm{s} . \mathrm{d} ., n=3$ ) of the matrix invaded cells in MEG3sh relative to the Ctrlsh. The $P$ values were calculated using Student's $t$-test (two-tailed, two-sample unequal variance), ${ }^{\star} P<0.05,{ }^{\star \star} P<0.01$. 
Table 1 | KEGG pathway analysis of the deregulated genes identified by microarray and RNA-sequencing after downregulation of MEG3 and EZH2 by siRNA in BT-549 cells using GeneSCF.

\begin{tabular}{|c|c|c|c|c|c|}
\hline \multirow[t]{2}{*}{ KEGG-ID } & \multirow[t]{2}{*}{ KEGG pathways } & \multicolumn{2}{|c|}{ MEG3 ( $P$ value) } & \multicolumn{2}{|c|}{ EZH2 (P value) } \\
\hline & & Microarray & RNA-seq & Microarray & RNA-seq \\
\hline hsa05200 & PI3K-Akt signalling pathway & 0.0000000307 & 0.00036 & 0.0001 & 0.00000376 \\
\hline hsa04390 & Proteoglycans in cancer & 0.000000472 & 0.00494 & 0.00000787 & 0.0021 \\
\hline hsa05166 & Pathways in cancer & 0.00000099 & 0.000000697 & 0.00000808 & 0.002 \\
\hline hsa04350 & TGF-beta signalling pathway & 0.0000059 & 0.0000893 & 0.00000062 & 0.0002 \\
\hline hsa05205 & HTLV-I infection & 0.00000865 & 0.00000239 & 0.0002 & 0.0432 \\
\hline hsa04151 & Focal adhesion & 0.0000188 & 0.00076 & 0.0005 & 0.0000239 \\
\hline hsa04910 & Insulin signalling pathway & 0.0000246 & 0.0175 & 0.0087 & 0.0274 \\
\hline hsa04510 & Hippo signalling pathway & 0.0000867 & 0.000002029 & 0.0001 & 0.0022 \\
\hline hsa01100 & Colorectal cancer & 0.0001 & 0.0017 & 0.000029 & 0.0009 \\
\hline hsa05414 & Regulation of actin cytoskeleton & 0.0003 & 0.0001 & 0.0012 & 0.0000114 \\
\hline hsa04010 & Endocytosis & 0.0005 & 0.0000187 & 0.0002 & 0.0002 \\
\hline hsa00532 & Pancreatic cancer & 0.0008 & 0.01 & 0.0002 & 0.0000439 \\
\hline hsa04668 & Hypertrophic cardiomyopathy & 0.0013 & 0.00000569 & 0.0053 & 0.0238 \\
\hline hsa05203 & Chagas disease & 0.0013 & 0.0028 & 0.000022 & 0.0054 \\
\hline hsa04520 & Adherens junction & 0.0016 & 0.0002 & 0.000079 & 0.0005 \\
\hline hsa05142 & Viral carcinogenesis & 0.0017 & 0.0144 & 0.0082 & 0.0177 \\
\hline hsa05410 & TNF signalling pathway & 0.0019 & 0.00107 & 0.0052 & 0.0001 \\
\hline hsa05212 & Glycosaminoglycan biosynthesis & 0.0027 & 0.002 & 0.0311 & 0.0136 \\
\hline hsa04144 & MAPK signalling pathway & 0.0062 & 0.0004 & 0.014 & 0.0001 \\
\hline hsa04810 & Dilated cardiomyopathy & 0.02 & 0.0000489 & 0.0013 & 0.0289 \\
\hline hsa05210 & Metabolic pathways & 0.04 & 0.0269 & 0.0009 & 0.0216 \\
\hline
\end{tabular}

target the multiple TGF- $\beta$ pathway genes in trans? For this purpose, we wanted to fine-map genome-wide MEG3 binding sites using the ChOP method with minor modifications. We have previously used ChOP methodology to characterize the Kcnq1ot1 IncRNA binding sites on mouse chromosome 7 (refs 10,35). This method is conceptually equivalent to other methods currently used to fine-map RNA binding sites ${ }^{16,17,19,36}$. We used 15 biotinlabelled antisense DNA oligonucleotides (oligos) spanning across MEG3 RNA (Supplementary Fig. 6a) to ensure robust capture of $M E G 3$ RNA-associated genomic loci with streptavidin beads. The ChOP pull-down using MEG3 antisense oligos detected specific enrichment of the MEG3 RNA, but not abundantly expressed nuclear-enriched MALAT1 lncRNA, whereas pull-down with a biotin probe against green fluorescence protein (GFP) RNA (with no known target in the human genome), used as a negative control, detected neither MEG3 nor MALAT1, highlighting the specificity of the ChOP pull-down assay (Fig. 4a). We then subjected the ChOP pull-down chromatin material with MEG3 and control probes to high-throughput DNA sequencing. By considering the MEG3-enriched regions over input and nonspecific GFP probes, we detected 6,837 MEG3-bound genomic regions associated with 5,622 genes (Table 2), as identified using the GREAT tool ${ }^{37}$. We found a significant overlap between the deregulated genes from the microarray experiment following MEG3 downregulation and the genes associated with MEG3 peaks (300 genes, $P<5 \mathrm{e}^{-14}$, hypergeometric distribution), indicating a functional role of the MEG3 peaks in the regulation of associated genes (Table 2, Supplementary Fig. 6b, Supplementary Data 8 and Supplementary Data 9). We also observed a significant overlap between the deregulated genes from RNA sequencing or from both microarray and RNA sequencing experiments, and the genes associated with MEG3 peaks (Supplementary Fig. 6b,c). When we performed network analysis with the 300 deregulated genes associated with the MEG3 peaks, we found that TGF- $\beta$ was one of the major affected pathways (Supplementary Fig. 7a,b). The majority of the MEG3-bound peaks associated with the deregulated genes were located distal to the promoter, including genes involved in the TGF- $\beta$ pathway (Table 2 , Fig. $4 \mathrm{~b}$ and Supplementary Fig. 8a-o), suggesting that the MEG3-bound regions may serve as distal regulatory elements, and that the $M E G 3 / E Z H 2$ functional interaction contributes to their regulation. To verify whether the enrichment of the MEG3bound regions is due to an artefact of direct interaction between the MEG3 genomic locus and the MEG3 peaks identified, we performed ChOP using sense and antisense oligos. We validated the enrichment of the MEG3 peaks associated with the TGF- $\beta$ pathway genes in ChOP pull-down with the antisense oligos but not with the sense oligos. The enrichment with antisense oligos was lost when the chromatin was pretreated with RNase A (Supplementary Fig. 9a). This further suggests that the pull-down with antisense oligos is mediated by MEG3 RNA rather than being the result of technical artefacts. To identify MEG3-bound peaks that overlap with putative enhancers in BT-549 cells, we performed H3K4mel ChIP-seq and overlapped the H3K4me1 peaks with MEG3 peaks, and found that 662 MEG3 peaks overlapped with $\mathrm{H} 3 \mathrm{~K} 4 \mathrm{me} 1$ peaks (H3K4me1/MEG3 peaks) in BT-549 cells (Table 2). The H3K4me1/MEG3 peaks' associated genes showed a significant overlap with the genes that were deregulated upon downregulation of $M E G 3$ and had at least one associated MEG3 peak (Table 2 and Supplementary Fig. 6c).

We observed a decrease in the enrichment of both H3K27me3 and EZH2 over the distal MEG3-bound peaks of the TGF- $\beta$ pathway genes upon downregulation of MEG3 (Fig. 4c and Supplementary Fig. 9b), suggesting that $M E G 3$ is required for PRC2 recruitment and $\mathrm{H} 3 \mathrm{~K} 27$ me3 maintenance at the distal regulatory elements. We tested the enhancer activity of the TGFBR1-associated H3K4me1/MEG3 peaks using the luciferase system and found a significant increase in the enhancer activity of the peaks in the MEG3 shRNA-transduced cells compared with the control shRNA cells (Supplementary Fig. 9c). We performed the chromosome conformation capture (3C) assay to measure the 


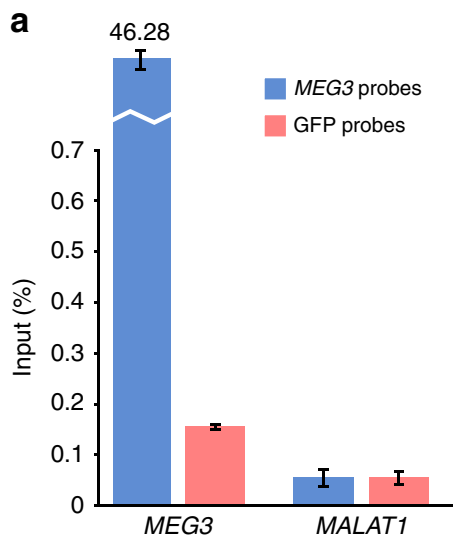

C

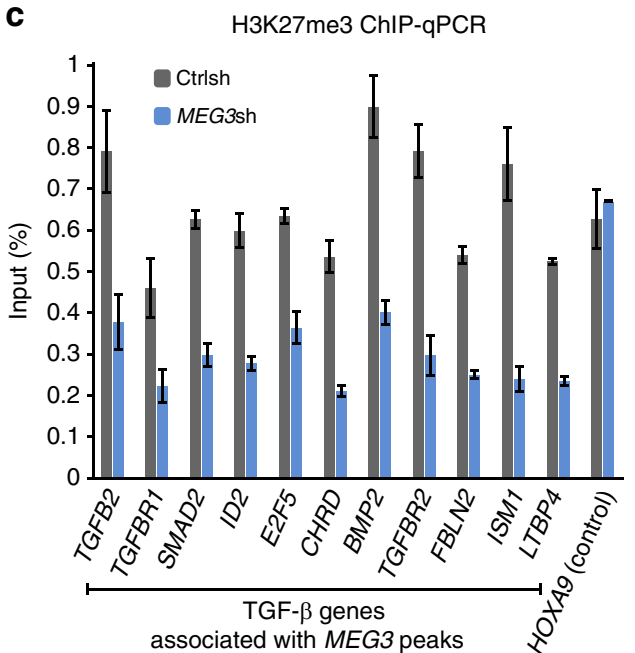

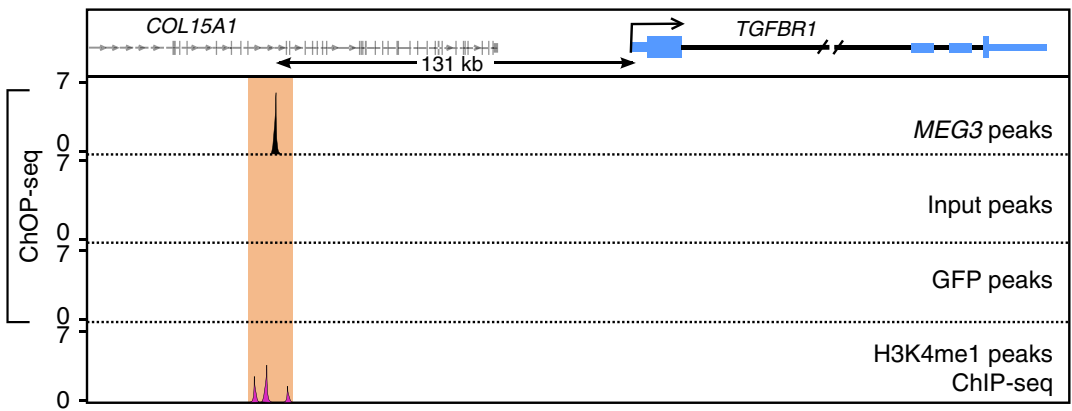

d

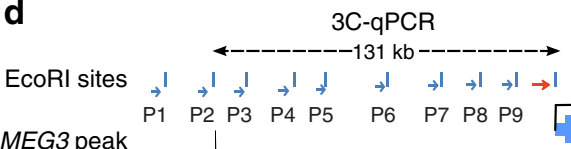

TGFBR1

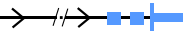

Figure 4 | Genome-wide mapping of MEG3 IncRNA binding sites. (a) RT-qPCR analysis showing specific enrichment (presented as percentage of input) of MEG3 but not MALAT1 RNA in the ChOP pull-down assay with MEG3 antisense probes. The ChOP pull-down with GFP antisense oligo, used as a negative control, did not show any enrichment of MEG3 and MALAT1 RNAs. (b) Genomic tracks showing ChOP-seq (MEG3, GFP and input) and ChIP-seq (H3K4me1) intensities, visualized in log scale. The MEG3 binding site is located upstream of the TGFBR1 gene (falls within the intron of the COL15A1 gene) and it overlaps with H3K4me1 peaks in BT-549 cells. (c) ChIP-qPCR showing enrichment of H3K27me3 chromatin marks, presented as percentage of input, over the MEG3 peaks associated with the TGF- $\beta$ genes in Ctrlsh and MEG3sh cells ( \pm s.d., $n=3$ ). (d) Schematic outline of the TGFBR1 gene showing MEG3 peaks and the location of 3 C primers (P1-P9), as indicated by arrows. EcoRI restriction sites are shown as blue vertical lines. Each error bar represents \pm s.d. from three experiments. Looping events between the upstream MEG3 binding site (corresponding to P2 primer) and the TGFBR1 promoter detected by $3 C$-qPCR in Ctrlsh and MEG3sh cells. The $P$ values were calculated using Student's $t$-test (two-tailed, two-sample unequal variance), ${ }^{\star} P<0.05$.

long-range interactions between the upstream $\mathrm{H} 3 \mathrm{~K} 4 \mathrm{me} 1 / \mathrm{MEG} 3$ peaks and the TGFBR1 promoter. In our 3C experiment, we detected interaction between the upstream H3K4me1/MEG3 peaks and the TGFBR1 promoter. Interestingly, these interactions were enhanced in the MEG3 shRNA-transduced cells compared with the control shRNA cells (Fig. 4d), indicating that the MEG3/ PRC2 functional interaction could regulate the activity of the distal regulatory elements.

MEG3 targets the TGF- $\beta$ pathway genes via GA-rich sequences. We next tried to investigate the mechanisms that facilitate how MEG3 lncRNA selects its target regions across the genome. First, we looked for common sequence motifs enriched in the MEG3-bound genomic regions and identified a strong GA-rich sequence motif that was overrepresented among the 6,837 MEG3 peak summits (motif $e$-value: $1.7 \mathrm{e}^{-976}$ ) (Fig. 5a). The GA-rich motif was also overrepresented among the 532 MEG3 peaks (motif $e$-value: $6.3 \mathrm{e}^{-904}$ ) associated with the MEG3-deregulated genes (Fig. 5a), suggesting that the GA-rich repeat may play a functional role in targeting of the MEG3 RNA to chromatin. Interestingly, by using the ChIRP technique, similar GA-enriched motifs were identified among the binding sites of the chromatinmodulating RNAs roX2 and HOTAIR, indicating that GA-enriched motifs may play an important role in the targeting of lncRNAs across the genome ${ }^{19}$. Previously, several studies using different techniques have shown that GA-rich homopurine sequences can form triplex structures ${ }^{38-40}$. Overrepresentation of GA-rich sequences among the genomic binding sites of the lncRNAs analysed (MEG3, HOTAIR and roX) raises the possibility that the lncRNAs may be recruited to their target genes via RNA-DNA triplex formation. By using Triplexator software $^{41}$ (which can predict triplex target sites, TrTS), we found a greater number of the predicted TrTS in the MEG3 peak summit ( $\pm 200 \mathrm{bp}$ from the centre of the peak) than the flanking sequences (200 bp upstream and $200 \mathrm{bp}$ downstream of the peak summit; Fig. 5b). Triplexator was also used to scan for triplexforming oligonucleotides (TFOs) within the MEG3 RNA, and several TFOs with high scores were detected. Interestingly, the TFOs with high scores are also enriched with GA-rich sequences 
(Table 3), indicating that the GA-rich sequences from target genes and MEG3 RNA could form triplex structures by forming Hoogsteen bonds between RNA and DNA. To test the ability of MEG3 lncRNA to form triplex structures, we used a 20nucleotide-long GA-rich RNA oligo (hereon referred to as MEG3 TFO) located at the $5^{\prime}$-end of the MEG3 RNA and its sequence overlap with the TFOs (TFO1, TFO2 and TFO3) with high score that were identified by Triplexator (Fig. $5 \mathrm{c}$ and Table 3). Using electrophoretic mobility shift assay, we tested the triplex-forming ability of the MEG3 TFO (single-stranded RNA, ssRNA) with the GA-rich (double-stranded DNA, dsDNA) $M E G 3$ peak sequences associated with the selected TGF- $\beta$ pathway target genes (TGFBR1, TGFB2 and SMAD2) in vitro (Fig. 5d). Consistent with the Triplexator predictions, we observed a shift in the end-labelled GA-rich dsDNA sequences when incubated with increasing concentrations of the MEG3 TFO, indicating triplex formation between the MEG3 TFO and the GA-rich MEG3 peak summits (Fig. 5d, compare lane 1 with lanes 2 and 3), but not with a control RNA oligo selected from the MEG3 lncRNA with no GA bias (Fig. 5d, compare lane 1 with lanes 8 and 9). The triplex structures were sensitive to RNase A treatment but were resistant to RNase $\mathrm{H}$ digestion (Fig. $5 \mathrm{~d}$, lanes 4 and 5, respectively), while an in vitro formed RNA-DNA hybrid was digested by RNase H (Supplementary Fig. 10a). These results together suggest that the observed shift was not because of Watson-Crick RNA-DNA pairing. We also observed that these shifts were affected when specific competitor (the same GA-rich dsDNA oligo, unlabelled) was used but were unaffected by nonspecific competitor (unlabelled control dsDNA oligo; Fig. 5d,

\begin{tabular}{|c|c|c|}
\hline $\begin{array}{l}\text { Summary of the MEG3 binding sites } \\
\text { in BT- } 549 \text { cells }\end{array}$ & $\begin{array}{c}\text { No. } \\
\text { of } \\
\text { peaks }\end{array}$ & $\begin{array}{c}\text { No. of } \\
\text { genes } \\
\text { associated }\end{array}$ \\
\hline MEG3 binding sites in BT-549 cells & 6,837 & 5,622 \\
\hline Number of MEG3 peaks in promoter & 217 & 173 \\
\hline Number of MEG3 peaks in promoter-distal region & 6,620 & 5,449 \\
\hline $\begin{array}{l}\text { Peaks associated with MEG3-deregulated genes } \\
\text { (MEG3-deregulated peaks) }\end{array}$ & 532 & 300 \\
\hline Number of MEG3-deregulated peaks in distal & 524 & 292 \\
\hline $\begin{array}{l}\text { Distal MEG3 peaks overlap with BT-549 } \\
\text { H3K } 4 \text { me1 peaks }\end{array}$ & 662 & 959 \\
\hline $\begin{array}{l}\text { Distal MEG3-derugulated peaks overlap } \\
\text { with BT-549 H3K4me1 signals }\end{array}$ & 56 & 52 \\
\hline
\end{tabular}

compare lane 6 with 7). We did not observe any complex formation between $M E G 3$ TFO incubated with end-labelled control DNA sequences (with no GA bias) or control RNA incubated with control DNA sequences (Supplementary Fig. 10b,c). To further check the specificity of the interaction between MEG3 TFO and GA-rich DNA sequences, we mutated the core sequences of the TGFBR1-associated MEG3 peak and found that triplex formation was compromised between the mutant TGFBR1 dsDNA oligo and the MEG3 TFO (Fig. 5e). These observations suggest that $M E G 3$ may be recruited to genomic loci through the formation of RNA-DNA triplex structures. We predicted the triplex-forming ability of GA-rich motifs of another chromatin-interacting lncRNA, HOTAIR, and found more Triplexator-predicted TrTS from the HOTAIR summit regions than from the neighbouring sequences (Supplementary Fig. 10d,e).

We then investigated the formation of RNA-DNA triplex structures by using an alternative method whereby biotin-labelled MEG3 TFO and a control RNA oligo were either used to transfect BT-549 cells (Fig. 5f) or incubated with nuclei isolated from BT-549 cells (Fig. 5g). Upon pull-down with streptavidin magnetic beads, we found significant enrichment of the selected MEG3 peaks associated with the TGF- $\beta$ genes-with MEG3 TFO compared with control oligo. The enrichment of the MEG3 target sequences was unaltered upon treatment with RNase $\mathrm{H}$, suggesting that the interaction of the MEG3 TFO with the target DNA sequence is not mediated by Watson-Crick RNA-DNA pairing (Fig. 5f,g). We next investigated whether the MEG3 TFO occupancy at the MEG3 target genes alters their transcriptional regulation. To this end, we analysed expression of the three key TGF- $\beta$ genes TGFB2, TGFBR1 and SMAD2 in BT-549 cells after transfection with MEG3 TFO or control RNA oligo. We found that expression of the TGF- $\beta$ pathway genes was marginally, but significantly, upregulated in the MEG3 TFO-transfected cells compared with the cells transfected with control oligo (Fig. 5h). These results indicate that the MEG3 TFO sequence can compete with endogenous full-length MEG3 RNA in binding to MEG3 target sites, thus affecting the endogenous function of MEG3 RNA.

We also performed circular dichroism (CD) spectroscopy to investigate the triplex-forming ability of some of the MEG3 target sites with MEG3 TFO. Figure 5i (left) shows the CD spectrum of the dsDNA oligo corresponding to the TGFB2-associated MEG3 peak incubated with $M E G 3$ TFO ssRNA and the corresponding spectrum with a control ssRNA. The spectrum of the MEG3 TFO sample has some distinct features, such as a distinct blue-shift ( $\sim 10 \mathrm{~nm}$ ) of the peak $\sim 270-280 \mathrm{~nm}$ and a strong negative peak at $\sim 210 \mathrm{~nm}$, which are not seen in the sample containing the control ssRNA. The effect is emphasized in the inset in Fig. 5i,

Figure 5 | MEG3 IncRNA regulates its target genes through triplex structure formation. (a) Predicted GA-rich motifs enriched in all MEG3 peaks and peaks associated with deregulated genes using MEME-ChIP. (b) Number of TrTS over the MEG3 peak summits and neighbouring regions, predicted by Triplexator $^{41}$. (c) The schematic shows the MEG3 TFO used in the triplex assays. The exons are colour-coded as described before. (d) Electrophoretic mobility shift assay. End-labelled dsDNA oligos (sequences provided in the schematic with gene name) were incubated alone (lane 1) or with increasing concentrations of MEG3 ssRNA TFO (lanes 2 and 3: shift indicated with arrow) or with increasing concentrations of control ssRNA oligo (lanes 8 and 9). dsDNA oligos were incubated with MEG3 TFO and treated with either RNase A (lane 4) or RNase H (lane 5). dsDNA oligos were incubated with MEG3 ssRNA TFO in the presence of either unlabelled specific competitor (lane 6) or nonspecific competitor (lane 7). (e) TGFBR1-associated MEG3 peak sequence and its mutated version (the changed nucleotides are in red) were incubated alone (lanes 1 and 7 ) or with MEG3 TFO. Arrow indicates complex formation. (f,g) Enrichment of MEG3 peak sequences using biotin- and psoralen-labelled MEG3 TFO. RNase H-treated lysates were used to capture the labelled MEG3 TFO using streptavidin beads. The enrichment of MEG3 peaks is presented as the ratio between MEG3 TFO and control oligo ( \pm s.d., $n=3$ ). (h) RT-qPCR analysis of gene expression in BT-549 cells transfected with either MEG3 TFO or control RNA oligo. Expression in MEG3 TFO presented relative to the control oligo ( \pm s.d., $n=3$ ). ${ }^{\star} P<0.05$, Student's $t$-test (two-tailed, two-sample unequal variance). (i) Left panel: $C D$ spectra of a 1:1 mixture of TGFB2 dsDNA and MEG3 TFO (ssRNA) are shown in black, and TGFB2 dsDNA and the control ssRNA are shown in red. Right panel: the sum of the individual CD spectra for TGFB2 dsDNA and MEG3 TFO (ssRNA) is shown in black, and the sum of the individual CD spectra for TGFB2 dsDNA and the control ssRNA is shown in red. Inset in the left and right panel shows the difference between the two spectra. 
where we show the difference in $\mathrm{CD}$ spectrum between the TGFB2 dsDNA oligo with the MEG3 TFO and the control ssRNA. These two features, and especially the strong negative peak at $\sim 210 \mathrm{~nm}$, are often seen for TFOs ${ }^{42-44}$. Figure $5 \mathrm{i}$ (right) shows the sum of the individual CD spectra for either TGFB2
dsDNA and MEG3 TFO (ssRNA) or TGFB2 dsDNA and the control ssRNA. For these artificial spectra, the difference between the MEG3 TFO and the control ssRNA is much smaller (Fig. $5 \mathrm{i}$, right, inset), supporting our conclusion that the change in $\mathrm{CD}$ spectrum when MEG3 TFO is incubated with the dsDNA TGFB2 a

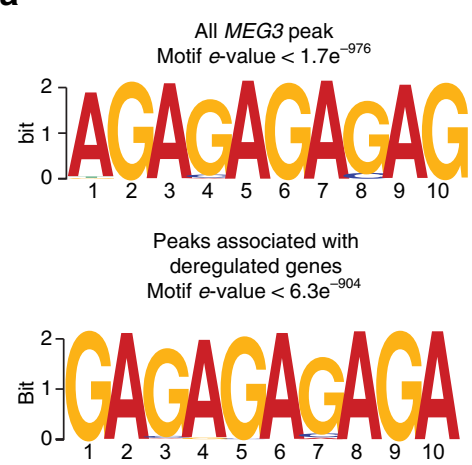

b

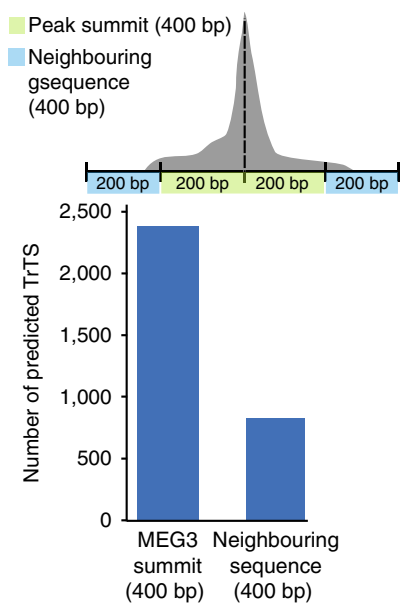

C

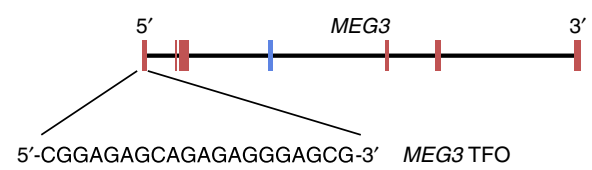

d

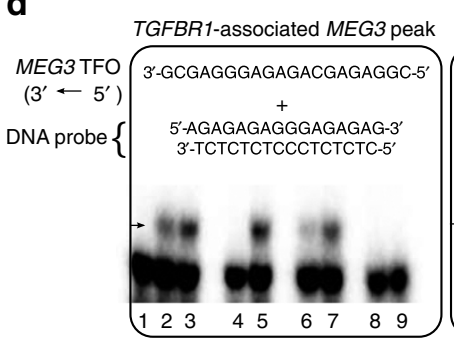

f

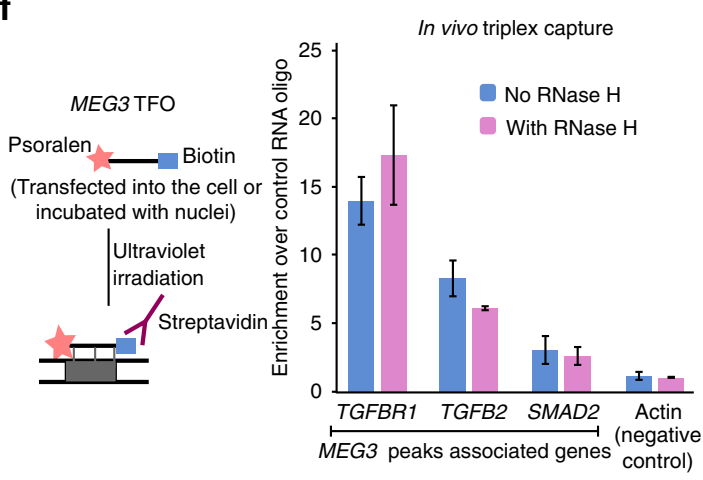

i

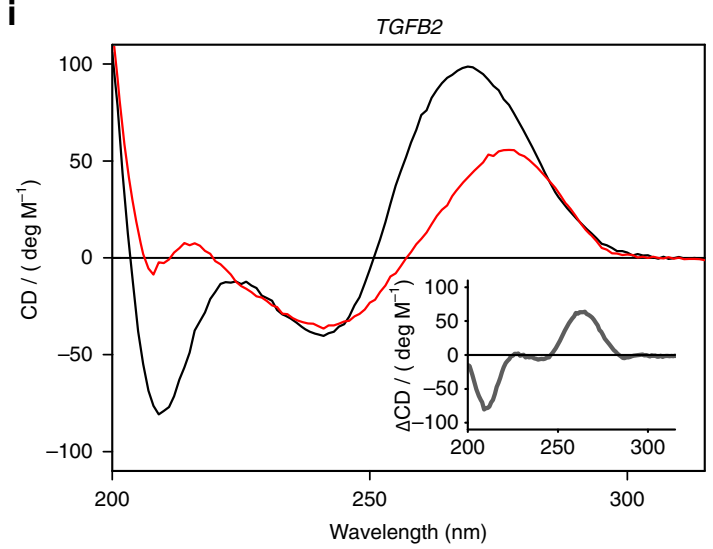

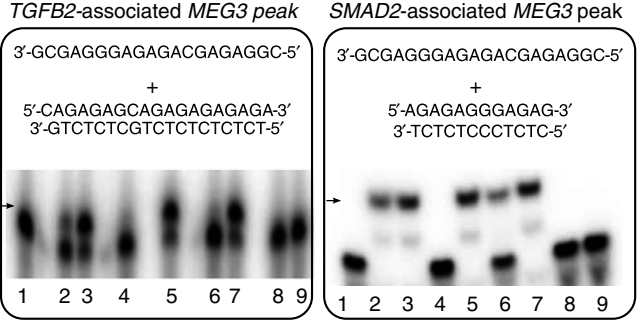
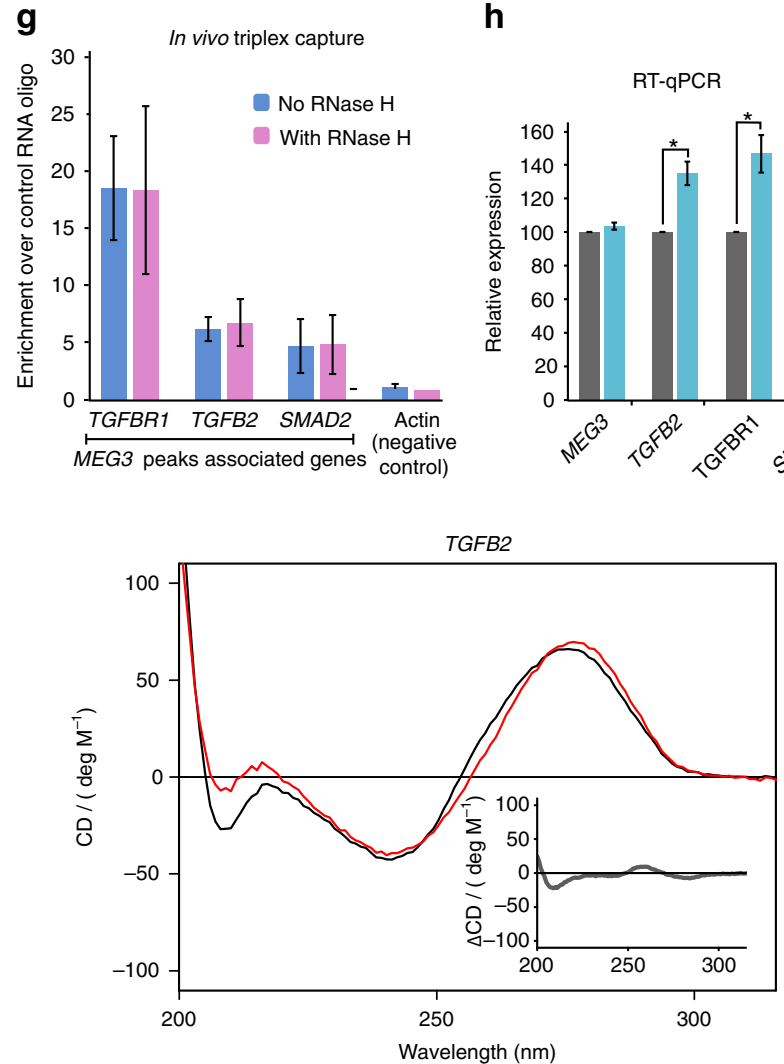

h

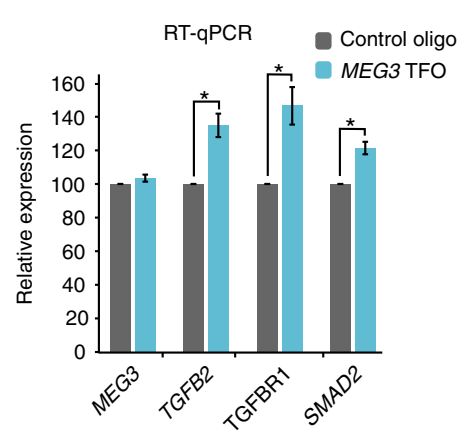

e

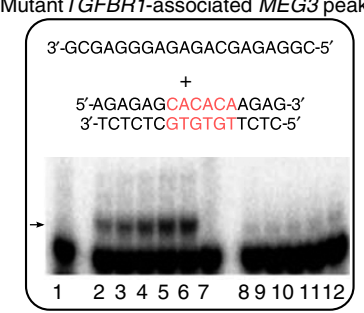

velength $(\mathrm{nm})$ 


\begin{tabular}{|c|c|c|}
\hline OligolD & TFOs $\left(5^{\prime}-3^{\prime}\right)$ & Score \\
\hline TFO1 & GGAGAGcAGAGAGGGAGcG & 18 \\
\hline TFO2 & GGcGGAGAGcAGAGAGGGAGcG & 19 \\
\hline TFO3 & AGAcGGcGGAGAGcAGAGAGGGAG & 21 \\
\hline TFO4 & AGGAtGGcAAAGGAtGAAGAGGA & 20 \\
\hline TFO5 & AAAtGAGAtAAAAGAGG & 15 \\
\hline TFO6 & GTcTTTGcTTGTGTT & 13 \\
\hline TFO7 & TGGGTGGGcTTcTGG & 13 \\
\hline TFO8 & TaGGGTTGTTGTGaG & 13 \\
\hline TFO9 & GGGcTGTTGTGaGGGG & 14 \\
\hline
\end{tabular}

TFOs, triplex-forming oligos; Score, triplex-forming potential scores.

The bold nucleotides indicate overlap with MEG3 TFO with high score.

is owing to a specific interaction between the two. We thus interpret the $\mathrm{CD}$ data, in combination with evidence from the complementary techniques, as being owing to the formation of a triplex structure between the dsDNA TGFB2 and the MEG3 TFO ssRNA. CD spectra similar to the ones in Fig. 5i (left) were also detected with the SMAD2- (Supplementary Fig. 11a) and TGFBR1 (Supplementary Fig. 11b)-associated MEG3 peaks, indicative of triplex formation.

Triplex structures are associated with TGF- $\beta$ genes in vivo. Our in vitro triplex formation assay with MEG3 RNA TFO and GA-rich MEG3 target sequences suggests that RNA-DNA triplex formation could guide $M E G 3$ lncRNA to its target genes across the genome. This raises an intriguing question as to whether RNA-DNA triplex structures are present in vivo. In order to identify such triplex structures in BT-549 cells, we wanted to perform immunostaining with anti-triplex dA.2rU antibody, which can detect triplex structures. The specificity of the dA.2rU antibody in detecting triplex structures has been verified by enzyme-linked immunosorbent assay ${ }^{45,46}$. In addition, anti-triplex dA.2rU antibody has been used in immunostaining to detect the triplex structures on polytene chromosomes, and also in two-cell early pre-implantation mouse embryos $^{45,47}$. Since the anti-triplex dA.2rU antibody was raised against the triplex derived from homopolymeric nucleic acids (poly $(\mathrm{rU}) \cdot \operatorname{poly}(\mathrm{dA}) \cdot \operatorname{poly}(\mathrm{rU})$ complex $)^{46}$, we wanted to test the ability of the anti-triplex dA.2rU antibody to recognize nonhomopolymeric triplexes, which would be relevant for detecting triplex structures in vivo. For this, we performed immunodots with DNA triplexes built from poly-purine/poly-pyrimidine sequences, including controls for the antibody reactivity. As expected, the antibodies recognized homopolymeric triplexes containing a poly $(\mathrm{dA})$ backbone and did not bind to nucleic acids when non-complementary sequences in solutions impeded formation of triple-stranded complexes. Also, the antibodies clearly bound to the triplex DNA made with poly-purine/polypyrimidine sequences (Supplementary Fig. 12a), indicating that antibody reactivity is not restricted to three-stranded configurations assembled with homopolymeric nucleic acids. To detect triplex structures in vivo, immunostaining was performed on BT-549 cells with anti- triplex dA.2rU antibody, and specific staining was observed with anti- triplex dA.2rU antibody (Fig. 6a). The anti-triplex staining was distributed in both the nucleus and the cytoplasm with more enrichment of the triplexspecific staining in the nuclear compartment (Fig. 6a). Triplexspecific staining was significantly reduced in the cells treated with RNase A (Fig. 6b, left and middle panel), but it was resistant to treatment with RNase $\mathrm{H}$, which specifically cleaves RNA-DNA hybrids (Fig. $6 \mathrm{~b}$, right panel). The triplex staining pattern in
BT-549 cells was similar to the staining of triplex structures that had been detected previously in human cells using a monoclonal anti-triplex antibody, Jel 318 (ref. 48 and Supplementary Fig. 12b). Although the triplex structures were more enriched in the nucleus, we detected triplex-specific staining in the cytoplasm, which could be due to recognition of the triplex structures present in mitochondria ${ }^{49,50}$. To test this, we labelled the mitochondria in BT-549 cells with MitoTracker followed by immunostaining with anti-triplex antibody. We indeed observed a co-localization of the mitochondrial staining with triplex signals from cytoplasm, suggesting that a part of the cytoplasmic triplex signals are contributed by the triplex structures present in mitochondria (Supplementary Fig. 12c). We next wanted to determine whether the triplex structures present at the MEG3 binding sites are associated with the TGF- $\beta$ pathway genes in BT549 cells. We performed triplex-ChIP with anti-triplex dA.2rU antibody and observed enrichment of the selected MEG3 peaks associated with the TGFBR1, TGFB2 and SMAD2 genes. To check the specificity of the anti-triplex dA.2rU pull-down, we pretreated the chromatin with either RNAse $\mathrm{H}$ or RNase A. RNase A treatment, but not RNase $\mathrm{H}$, treatment resulted in complete loss of triplex enrichment (Fig. 6c). We also performed triplex-ChIP in MEG3-downregulated BT-549 cells and found a decrease in the enrichment of the triplex structures over the $M E G 3$ peaks associated with the TGFBR1, TGFB2 and SMAD2 genes, suggesting that the $M E G 3$ lncRNA regulate these genes through triplex formation (Fig. 6d).

Chromatin-binding region of MEG3 is functionally distinct. We wanted to determine whether the MEG3 RNA sequences required for the PRC2 interaction and RNA-DNA triplex formation are functionally distinct. For this purpose, we generated a MEG3 mutant by deleting the core MEG3 TFO (the TFO used in the triplex assay above) containing GA-rich sequences and named this $\triangle 46-56$ MEG3 (46-56 indicates the position of the nucleotides with respect to the $5^{\prime}$-end of MEG3). We found that the TFO deletion had no effect on the interaction of PRC2 with MEG3 (Fig. 7a). Considering that $\triangle 345-348$ MEG3 affects the PRC2 interaction of WT MEG3 (Figs 7a and 2e), we decided to test the chromatin-interacting property of the WT and MEG3 RNAs with deletions ( $\triangle 46-56$ MEG3 and $\triangle 345-348$ MEG3) by transfecting BT-549 cells with in vitro-synthesized biotin-labelled WT or mutant MEG3 RNAs ( $\triangle 46-56$ MEG3 or $\triangle 345-348$ MEG3). We found that the WT and $\triangle 345-348$ MEG3 RNAs could pulldown MEG3 peak sequences associated with the TGF- $\beta$ genes (TGFB2, TGFBR1 and SMAD2), but not when the pull-down was performed with the $\triangle 46-56$ MEG3 TFO deletion (Fig. 7b). This suggests that the decrease in the association between MEG3 and PRC2 does not have any effect on the chromatin-binding property of MEG3 RNA. Taken together, these observations indicate that the chromatin targeting and PRC2 interaction properties of MEG3 lncRNA are mediated by distinct RNA sequences (Fig. 7c).

\section{Discussion}

Previous studies have identified thousands of $\operatorname{lncRNAs}$ that interact with repressive chromatin modifiers such as EZH2 (refs 24,25). The interaction of lncRNAs with chromatin modifiers suggests that lncRNAs may have a role in targeting the chromatin modifiers to chromatin. LncRNA-mediated recruitment of the chromatin modifiers to chromatin is exemplified by Kcnq1ot1 and HOTAIR lncRNAs, which have been shown to interact with chromatin and recruit repressive chromatin modifier EZH2 (refs 10,19,23,51). This raises the possibility that there could be many more lncRNAs that interact with chromatin and serve as a link between chromatin and chromatin modifiers. Use of 
a
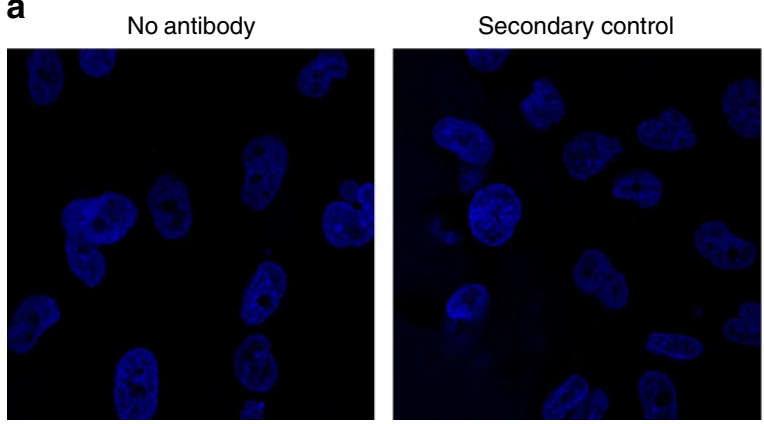

b
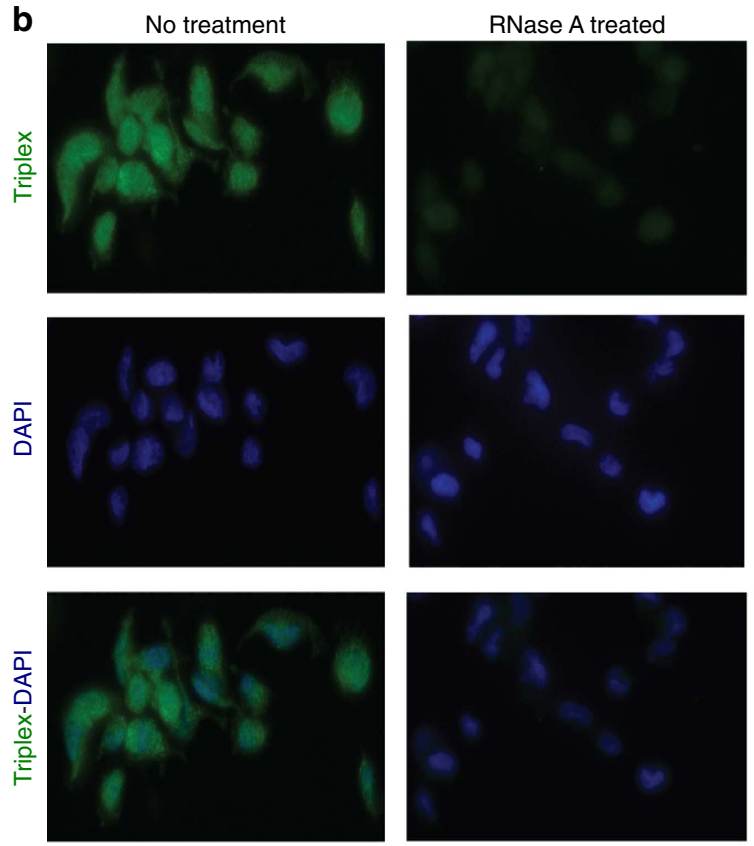

C

Triplex Chip-qPCR

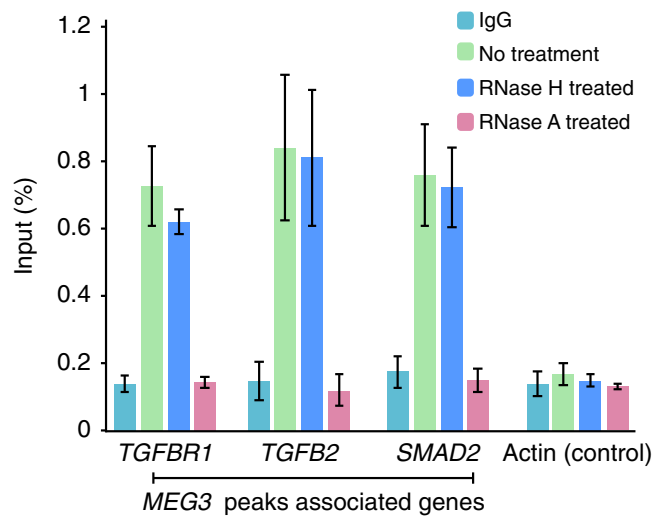

d
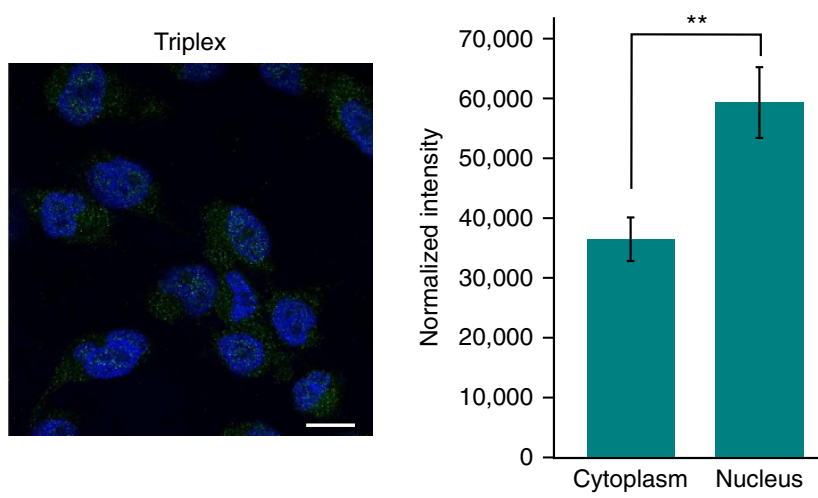

RNase $\mathrm{H}$ treated
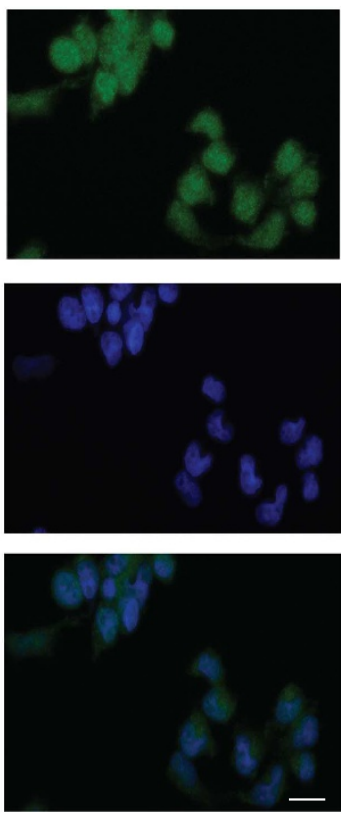

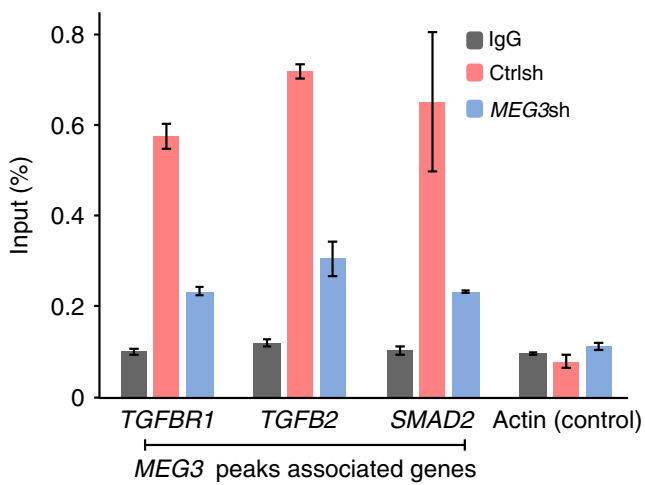

Figure 6 | RNA-DNA triplexes are present in vivo. (a) Confocal microscopic images showing immunostaining with anti-triplex dA.2rU antibody (green) in BT-549 cells. The nucleus is stained with DAPI (4,6-diamidino-2-phenylindole; blue). Immunostaining with no antibody and secondary antibody were used

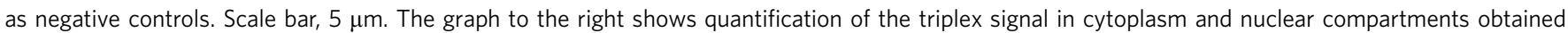
from the three-dimensional confocal images. The graph represents the average of cytoplasmic and nuclear signals from $>50$ cells in several microscopic fields. The error bars indicate s.e.m. The $P$ value was calculated using Student's $t$-test ${ }^{\star} P<0.01$. (b) RNA-DNA triplex structures are sensitive to RNase A but are resistant to RNase $\mathrm{H}$ in vivo. Top panel: immunofluorescent staining of BT-549 cells with anti-triplex dA.2rU antibody (green) with no treatment (left), pretreated with RNase A (centre), or pretreated with RNase H (right) as indicated. Middle panel: cells were counterstained with DAPI (blue). Bottom panel: overlay of the triplex signals with DAPI staining. Scale bar, $5 \mu \mathrm{m}$. (c) Triplex-ChIP-qPCR showing enrichment (presented as percentage of input) of triplex structures over the MEG3 peaks associated with the TGF- $\beta$ pathway genes (TGFBR1, TGFB2 and SMAD2) in BT-549 cells ( \pm s.d., $n=3$ ). Actin was used as a negative control. Chromatin was pretreated with RNase A or RNase H before ChIP. Immunoglobulin G (IgG) was used as an antibody control. (d) Triplex-ChIP-qPCR showing enrichment (presented as percentage of input) of triplex structures over the MEG3 peaks associated with the TGF- $\beta$ pathway genes (TGFBR1, TGFB2 and SMAD2) in Ctrlsh and MEG3sh BT-549 cells ( \pm s.d., $n=3$ ). IgG was used as an antibody control. 
a
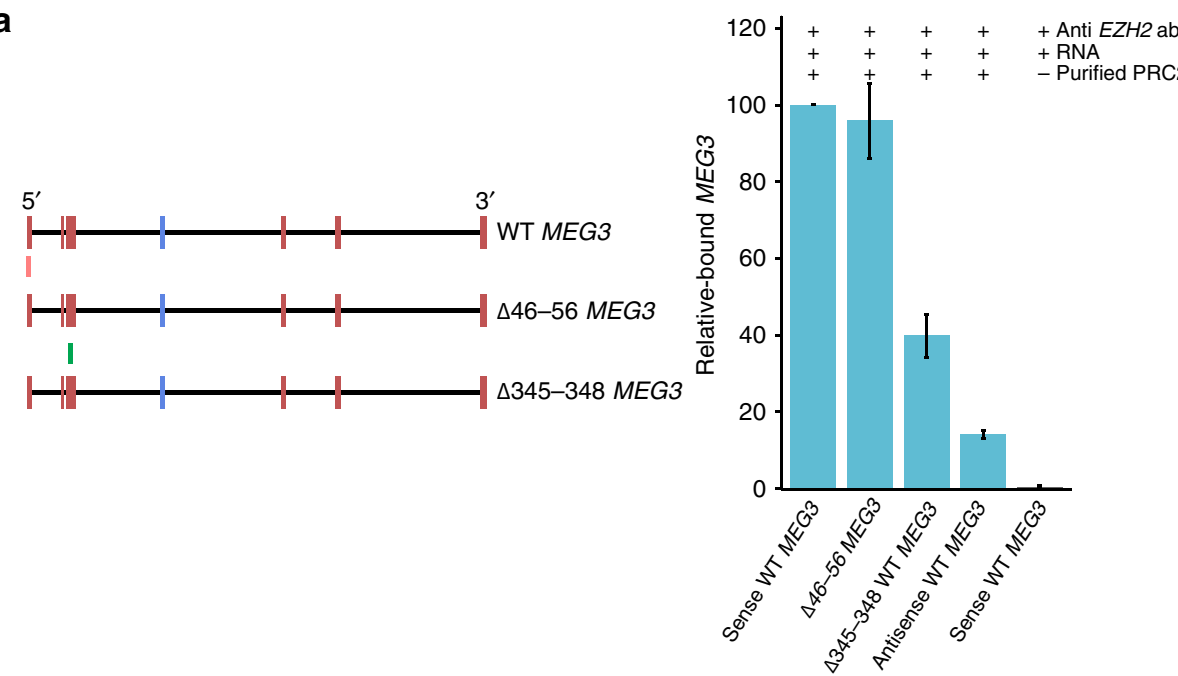

b
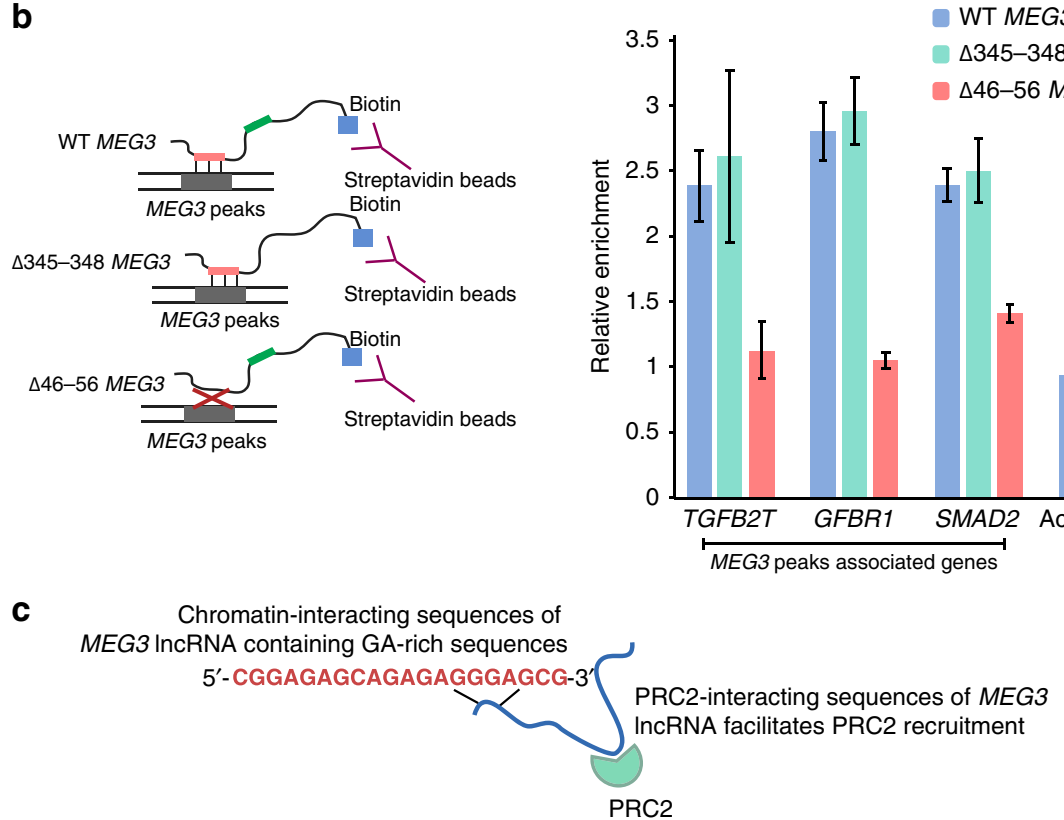

Chromatin-interacting sequences guide

MEG3 IncRNA to GA-rich DNA sequences by RNA-DNA triplex formation

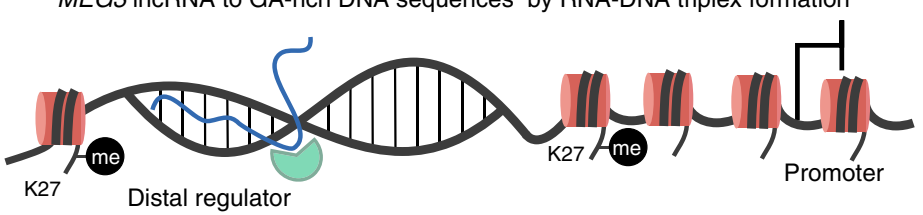

C

Chromatin-interacting sequences of MEG3 IncRNA containing GA-rich sequences
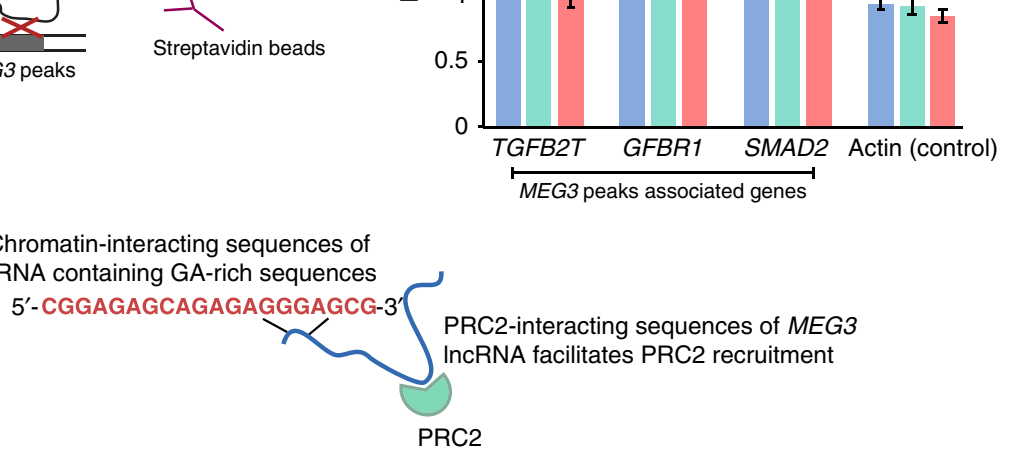

MEG3
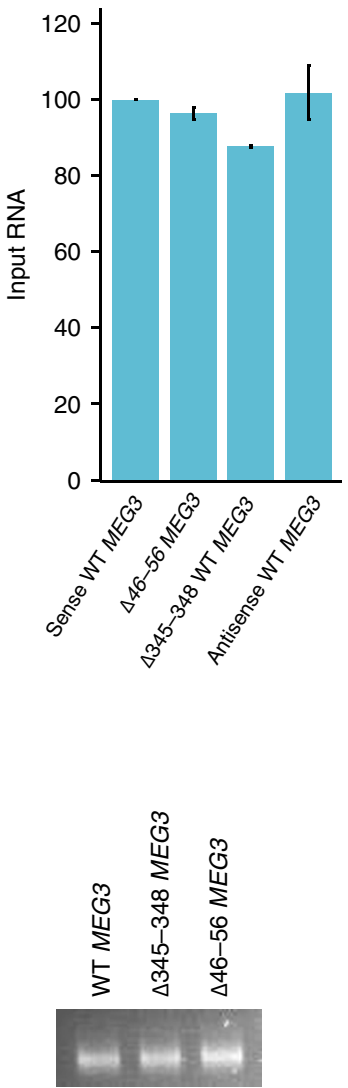
antibodies to EZH2 and its catalysed repressive chromatin mark H3K27me3 in our ChRIP-seq enabled us to identify 276 chromatin-interacting lncRNAs that are enriched in both EZH2 and H3K27me3 purified chromatin fractions. We expect that these chromatin-interacting $\operatorname{lncRNAs}$ will be a valuable resource for future investigations aimed at understanding the molecular mechanisms that dictate association of IncRNAs with chromatin. Indeed, by using one of the repressive chromatin-interacting IncRNAs, MEG3, we have characterized the mechanisms by which MEG3 IncRNA is guided to chromatin. Deciphering of the mechanisms that guide the EZH2-interacting lncRNAs to repressive chromatin will also shed light on how the PRC2 complex is targeted across the genome in an RNA-dependent manner. Repressive chromatin-associated lncRNAs also include several IncRNAs (PCA3, GAS6-AS1, CECR7 and BDNF-AS1) that have been implicated in cancer or other cellular functions, and among this BDNF-AS1 lncRNA has been shown to regulate gene expression by recruiting PRC2 (refs 30,52-54).

Mapping of the MEG3 binding sites across the genome in BT-549 cells revealed that the majority of MEG3 target sites are located distal to the promoter regions. Interestingly, a significant proportion of the promoter-distal MEG3 binding sites are enriched with enhancer chromatin marks. More importantly, we observed that the interaction between the TGFBR1 gene promoter and a putative enhancer increased significantly in the absence of MEG3. In addition, the downregulation of MEG3 also resulted in loss of EZH2 and H3K27me3 enrichment at the putative enhancer. These results suggest that the MEG3 lncRNA modulates the activity of the putative enhancer by regulating chromatin structure, thereby fine-tuning gene expression.

We detected over 6,800 MEG3 binding sites, and a proportion of these MEG3 peaks were associated with the genes that were either upregulated or downregulated after MEG3 knockdown in BT-549 cells. Given the association of MEG3 with a repressive chromatin modifier, EZH2, one would expect that the upregulated genes are more direct targets of the MEG3/EZH2 interaction. On the other hand, MEG3 peaks also flanked the genes that showed repression in the absence of MEG3. We suggest that the expression of these genes may be facilitated by the recruitment of EZH2 by MEG3, and this EZH2-dependent activation of genes was evident in recent findings where EZH2 has been shown to act as a coactivator of gene expression in prostate and breast cancer cells ${ }^{55,56}$. Although $M E G 3$-regulated genes were enriched in the MEG3 peaks, there was no one-to-one correlation between the MEG3 peaks and associated genes. This could be explained in part by the non-reversible nature of noncoding RNA-mediated chromatin modification; that is, once a chromatin mark is established in an RNA-dependent manner, it is maintained in the absence of the $\mathrm{RNA}^{57}$. The stable maintenance of RNA-mediated repressive modification after establishment was also observed with Kcnq1ot1 IncRNAmediated silencing, where the RNA was removed conditionally after the repressive chromatin was established ${ }^{58}$. It is possible that a similar mechanism exists in trans-acting lncRNAs such as $M E G 3$, where RNAi-mediated knockdown of the lncRNA does not lead to deregulation of all its target genes because of RNA-independent maintenance of the chromatin structure.

We have explored the molecular mechanisms by which MEG3 lncRNA contributes to regulation of the TGF- $\beta$ pathway. We found that the genes of the TGF- $\beta$ pathway are direct targets of $M E G 3$, and that it regulates these genes by binding to promoterdistal regulatory regions. Consistent with our results, a recent investigation has found that $M E G 3$ expression was downregulated upon TGF- $\beta 1$ treatment in human hepatic stellate cells, and that MEG3 overexpression inhibited the TGF- $\beta 1$-stimulated cell proliferation and induced apoptosis ${ }^{59}$. These observations together with our data indicate the existence of a probable feedback loop between the MEG3 and TGF- $\beta$ pathway. This target recognition by MEG3 occurs via triplex formation between GA-rich sequences of target genes and GA-rich sequences within MEG3 IncRNA. Interestingly such as MEG3, the HOTAIR binding sites also have GA-rich sequences ${ }^{19}$. Formation of triplex structures between MEG3 lncRNA and GA-rich sequences in our triplex assays indicates that GA-rich sequences may guide lncRNAs to their target genes. Immunostaining with monoclonal antibody to triplex structures revealed that these structures are widespread in vivo. Furthermore, using TriplexChIP assay, we found that triplex structures were present in vivo over the MEG3 peaks associated with the TGF- $\beta$ pathway genes. Taken together, these observations further suggest that targeting of MEG3 lncRNA to chromatin occurs through RNA-DNA triplex formation. Our observation on the mode of the chromatin targeting of MEG3 through RNA-DNA triplex formation along with the previous evidence of triplex-mediated communication of lncRNAs with their target genes suggests that this type of mechanism may be more general ${ }^{38,60-63}$. Similar to the transacting role of human MEG3 in breast cancer cells, a recent investigation by Kaneko et al. ${ }^{29}$ demonstrated that interaction between JARID2 and MEG3 lncRNA is critical for targeting of PRC2 complexes to multiple genes in trans in mouse embryonic stem cells. As JARID2 has also been found to play a critical role in activating the catalytic function of PRC2 by weakening the RNAPRC2 interaction, it would be interesting to investigate whether JARID2 has any such role in regulation of human MEG3-PRC2 interaction as well ${ }^{64}$. In this context, our study is particularly significant, as it contributes to our understanding of the mechanisms underlying the lncRNA-mediated targeting of PRC2 complex across the genome.

Functional overlap between MEG3- and EZH2-deregulated genes, and mapping of a significant number of $M E G 3$ binding sites to MEG3-deregulated genes indicate that MEG3 has a functional role in guiding PRC2 to its target genes across the genome. Fine-mapping of MEG3 RNA sequences required for PRC2 interaction and chromatin targeting via triplex formation suggests that while the triplex-forming sequences may guide MEG3 lncRNA to chromatin, the PRC2-interacting sequences facilitate the recruitment of PRC2 to promoter-distal regulatory regions, thereby depositing $\mathrm{H} 3 \mathrm{~K} 27 \mathrm{me} 3$ to modulate transcriptional activity (Fig. 7c). Our data on MEG3 RNA together with the published data on HOTAIR indicate that the GA-rich homopurine motif may be the preferred binding site for both MEG3 and HOTAIR IncRNAs. Interestingly, the GA-rich motif is also present in Drosophila ${ }^{65,66}$, Arabidopsis ${ }^{67}$ and mammalian ${ }^{68,69}$ polycomb response elements. In Drosophila melanogaster, PRC2 recruitment to the GA-rich motif has previously been shown to occur via a DNA-binding transcription factor, but no such factor has been characterized in mammals ${ }^{65}$. GA-rich motifs may be preferred sequences for RNA-dependent PRC2 recruitment, and thus lncRNAs may bypass the requirement for protein factors in PRC2 recruitment.

\footnotetext{
Methods

Molecular cloning. Full-length MEG3 cDNA was amplified from BT-549 nuclear RNA and cloned into either pCMV6-XL5 (OriGene) or pREP4 episomal vector (Life Technologies) using the primers described in Supplementary Data 10. The details of the exon compositions of the full-length $M E G 3$ clone are provided in the Results section. Mutant MEG3 RNAs ( $\triangle 340-348, \Delta 345-348$ and $\triangle 46-56$ MEG3) described in the manuscript were generated using the Quik-change site-directed mutagenesis kit (Agilent Technologies). Primers used in the site-directed mutagenesis and for cloning of MEG3 are provided in Supplementary Data 10. For in vitro transcription of biotin-labelled and unlabelled MEG3 RNA, full-length MEG3 (referred to as WT MEG3) or the mutant MEG3 RNAs, carrying various deletions, were cloned into pGEM-T Easy vector (Promega).
} 
Transfection and RT-qPCR assay. siRNAs were used for transfection using Lipofectamine RNAiMAX reagent (Life Technologies). For each siRNA transfection, 50,000 BT-549 or HF cells were seeded per well in 24-well plates $12-16 \mathrm{~h}$ before transfection. siRNAs against $M E G 3$ and $E Z H 2$ or the control siRNA were used for transfection at a final concentration of $65 \mathrm{nM}$ (details of siRNAs are provided in Supplementary Data 10). Forty-eight hours after transfection, RNA was isolated using the ReliaPrep RNA isolation kit (Promega). DNase I-treated RNA (500 ng) was converted to cDNA using reverse transcriptase (Promega) and assayed for gene expression by SYBR Green-based RT-qPCR using the Vii7 RealTime PCR system (Life Technologies) with the relevant primers listed in Supplementary Data 10. A negative control reaction without any cDNA was included in every qPCR. Episomal pREP4 plasmids (only pREP4 vector or pREP4 containing MEG3) were used to transfect BT-549 cells using Lipofectamine 2000 reagent (Life Technologies) and overexpression of MEG3 RNA was verified by RTqPCR $48 \mathrm{~h}$ after transfection, using primer pairs overlapping $M E G 3$ exon 3 (primer sequences are provided in Supplementary Data 10). pCAG-hEZH2 or the control DNA plasmid was used to transfect BT-549 cells using Lipofectamine 2000. EZH2 overexpression was confirmed by RT-qPCR $48 \mathrm{~h}$ after transfection, using primer pairs provided in Supplementary Data 10.

Cell culture and generation of stable clones. BT-549 cells were maintained in RPMI (Sigma) supplemented with 10\% fetal bovine serum (FBS) (Sigma). MDAMB-231 and HF cells were maintained in DMEM (Invitrogen) with 10\% FBS. BT549 cells were procured from CLS cell line service. MDA-MB-231 cells were kindly gifted by Dr Briegel Karoline (University of Miami Miller School of Medicine, Miami, USA) and HF cells were kindly gifted by Dr Bengt Westermark (Uppsala University, Uppsala, Sweden). MISSION lentiviral transduction particles expressing non-target shRNA control or shRNA against MEG3 were obtained from Sigma (the sequences are provided in Supplementary Data 10) and were used for transduction following the manufacturer's protocol. BT-549 cells $\left(5 \times 10^{4}\right)$ were plated in the wells of 24-well plates and transduced with either control or MEG3 shRNA lentiviral particles. Lentivirus-transduced BT-549 cells were selected with puromycin to obtain stably integrated shRNA vectors. The stable clones were maintained thereafter in RPMI containing puromycin $\left(1 \mu \mathrm{g} \mathrm{ml}^{-1}\right)$. We verified the downregulation of the MEG3 IncRNA in lentivirus-transduced stable clones by RTqPCR. Stable clones of MDA-MB-231 cells containing pREP4MEG3 were generated after antibiotic selection with hygromycin $\left(850 \mu \mathrm{g} \mathrm{ml}^{-1}\right)$, and maintained in DMEM containing hygromycin $\left(850 \mu \mathrm{g} \mathrm{ml}^{-1}\right)$. We verified the overexpression of the MEG3 RNA in MDA-MB-231 stable clones by RT-qPCR following the same procedure as described above.

ChRIP-seq. ChRIP was performed using BT-549 cells adapting the protocols from Mondal et al. ${ }^{28}$ and Kuo et al. ${ }^{70}$ with the modifications as detailed below. BT-549 cells were plated on tissue culture dish (7-8 million cells per $150 \mathrm{~mm}$ plate) and incubated overnight (14-16 h) with $4 \mathrm{sU}$ (Sigma) at a final concentration of $100 \mu \mathrm{M}$ in cell culture media. Next day, ActD $(5 \mu \mathrm{gml})$ was added to the media and incubated for 40-45 mins. To check the efficacy of ActD treatment, cells were incubated with and without ActD, and RNA was extracted and assayed level of $c-M y c$ RNA, with a short half-life, by RT-qPCR. After ActD incubation, cells were washed two times with PBS and crosslinked with $1 \%$ formaldehyde for 10 min with gentle shaking. Crosslinking was stopped by adding glycine to a final concentration of $125 \mathrm{mM}$ and incubated for $5 \mathrm{~min}$ with gentle shacking. Cells were subsequently washed twice with PBS followed by crosslinking on ice with ultraviolet. Cells were removed by scraping from the plate and resuspended in cold PBS. Nuclei were isolated using $1 \times$ nuclei isolation buffer $(40 \mathrm{mM}$ Tris- $\mathrm{HCl}(\mathrm{pH} 7.5)$,

$20 \mathrm{mM} \mathrm{MgCl}_{2}, 4 \%$ Triton X-100 and $1.28 \mathrm{M}$ sucrose) and washed again with PBS. The isolated crosslinked nuclei were resuspended in lysis buffer $(0.1 \%$ SDS, $0.5 \%$ Triton X-100, $20 \mathrm{mM}$ Tris- $\mathrm{HCl}$ ( $\mathrm{pH} 7.5), 150 \mathrm{mM} \mathrm{NaCl}$ and $1 \mathrm{ml}$ lysis buffer per 10 million of cells) supplemented with RNasein (Promega) and subjected to sonication (Bioruptor, 20-30 cycles) to obtain chromatin fragments of $\sim 1 \mathrm{~kb}$. An amount of $50-60 \mu \mathrm{g}$ of soluble chromatin was used in each chromatin immunoprecipitation and incubated with $5 \mu \mathrm{g}$ of anti-EZH2 (active motif) and anti-H3K27me3 (MerckMillipore) antibody. Antibody-bound chromatin was washed according to our earlier published protocol with buffers and was supplemented with RNasein. Protein A magnetic beads bound to immunoprecipitated chromatin were resuspended in $10 \times$ volume of elution buffer $(100 \mathrm{mM} \mathrm{NaCl}, 10 \mathrm{mM}$ Tris (pH 7.5), $1 \mathrm{mM}$ EDTA and $0.5 \%$ SDS) containing proteinase K. Proteinase $\mathrm{K}$ treatment was carried out at $55^{\circ} \mathrm{C}$ for $45 \mathrm{~min}$ followed by heating at $95^{\circ} \mathrm{C}$ for $10 \mathrm{~min}$ to reverse crosslinking. Chromatin-bound RNA was extracted with Trizol (Life Technologies) and subjected to DNase I (Promega) treatment to remove traces of DNA. Since the chromatin-bound RNA yield from one single experiment is suboptimal for high-throughput sequencing, we pooled RNA from 6 to 8 ChRIP pull-downs, and the sequencing library was made using SOLiD Total RNA-Seq Kit and sequenced the library using SOLiD platform (Applied Biosystem). SOLiD Total RNA-Seq Kit allows obtaining strand-specific RNA sequencing information. Since library preparation in SOLID protocol involves ligation of RNA adaptors to the RNA fragments by RNA ligase, no contamination from DNA fragments is expected. For input, nuclear RNA was isolated from 4sU- and ActD-treated BT-549 cells, and depleted ribosomal RNA using RiboMinus Eukaryote System v2 (Life Technologies). For ChRIP validation, we followed the same protocol as described above with or without ActD treatment using antibodies against H3K27me3, EZH2, H3K4me2 (Merck-Millipore) and nonspecific Rabbit IgG (immunoglobulin G; Merck-Millipore).

\section{References}

1. Kretz, M. et al. Control of somatic tissue differentiation by the long non-coding RNA TINCR. Nature 493, 231-235 (2013).

2. Klattenhoff, C. A. et al. Braveheart, a long noncoding RNA required for cardiovascular lineage commitment. Cell 152, 570-583 (2013).

3. Cesana, M. et al. A long noncoding RNA controls muscle differentiation by functioning as a competing endogenous RNA. Cell 147, 358-369 (2011).

4. Mohammad, F., Mondal, T., Guseva, N., Pandey, G. K. \& Kanduri, C. Kcnq1ot1 noncoding RNA mediates transcriptional gene silencing by interacting with Dnmt1. Development 137, 2493-2499 (2010).

5. Sun, L. et al. Long noncoding RNAs regulate adipogenesis. Proc. Natl Acad. Sci. USA 110, 3387-3392 (2013).

6. Pandey, G. K. et al. The risk-associated long noncoding RNA NBAT-1 controls neuroblastoma progression by regulating cell proliferation and neuronal differentiation. Cancer Cell 26, 722-737 (2014)

7. Cabianca, D. S. et al. A long ncRNA links copy number variation to a polycomb/trithorax epigenetic switch in FSHD muscular dystrophy. Cell 149, 819-831 (2012).

8. Zhao, J., Sun, B. K., Erwin, J. A., Song, J. J. \& Lee, J. T. Polycomb proteins targeted by a short repeat RNA to the mouse X chromosome. Science 322, 750-756 (2008).

9. Gupta, R. A. et al. Long non-coding RNA HOTAIR reprograms chromatin state to promote cancer metastasis. Nature 464, 1071-1076 (2010).

10. Pandey, R. R. et al. Kcnqlotl antisense noncoding RNA mediates lineagespecific transcriptional silencing through chromatin-level regulation. Mol. Cell 32, 232-246 (2008)

11. Hacisuleyman, E. et al. Topological organization of multichromosomal regions by the long intergenic noncoding RNA Firre. Nat. Struct. Mol. Biol. 21, 198-206 (2014).

12. Prensner, J. R. et al. The long noncoding RNA SChLAP1 promotes aggressive prostate cancer and antagonizes the SWI/SNF complex. Nat. Genet. 45, 1392-1398 (2013).

13. Tsai, M. C. et al. Long noncoding RNA as modular scaffold of histone modification complexes. Science 329, 689-693 (2010).

14. Ng, S. Y., Bogu, G. K., Soh, B. S. \& Stanton, L. W. The long noncoding RNA RMST interacts with SOX2 to regulate neurogenesis. Mol. Cell 51, 349-359 (2013).

15. Sun, S. et al. Jpx RNA activates Xist by evicting CTCF. Cell 153, 1537-1551 (2013).

16. Yang, L. et al. lncRNA-dependent mechanisms of androgen-receptor-regulated gene activation programs. Nature 500, 598-602 (2013).

17. Engreitz, J. M. et al. The Xist lncRNA exploits three-dimensional genome architecture to spread across the X chromosome. Science 341, 1237973 (2013).

18. Simon, M. D. et al. High-resolution Xist binding maps reveal two-step spreading during X-chromosome inactivation. Nature 504, 465-469 (2013).

19. Chu, C., Qu, K., Zhong, F. L., Artandi, S. E. \& Chang, H. Y. Genomic maps of long noncoding RNA occupancy reveal principles of RNA-chromatin interactions. Mol. Cell 44, 667-678 (2011).

20. Gardner, K. E., Allis, C. D. \& Strahl, B. D. Operating on chromatin, a colorful language where context matters. J. Mol. Biol. 409, 36-46 (2011).

21. Margueron, R. et al. Role of the polycomb protein EED in the propagation of repressive histone marks. Nature 461, 762-767 (2009).

22. Hansen, K. H. \& Helin, K. Epigenetic inheritance through self-recruitment of the polycomb repressive complex 2. Epigenetics 4, 133-138 (2009).

23. Kanduri, C. Kcnqlot1: a chromatin regulatory RNA. Semin. Cell Dev. Biol. 22, 343-350 (2011)

24. Zhao, J. et al. Genome-wide identification of polycomb-associated RNAs by RIP-seq. Mol. Cell 40, 939-953 (2010).

25. Khalil, A. M. et al. Many human large intergenic noncoding RNAs associate with chromatin-modifying complexes and affect gene expression. Proc. Natl Acad. Sci. USA 106, 11667-11672 (2009).

26. Guil, S. et al. Intronic RNAs mediate $\mathrm{EZH} 2$ regulation of epigenetic targets. Nat. Struct. Mol. Biol. 19, 664-670 (2012).

27. Kaneko, S., Son, J., Shen, S. S., Reinberg, D. \& Bonasio, R. PRC2 binds active promoters and contacts nascent RNAs in embryonic stem cells. Nat. Struct. Mol. Biol. 20, 1258-1264 (2013).

28. Mondal, T., Rasmussen, M., Pandey, G. K., Isaksson, A. \& Kanduri, C. Characterization of the RNA content of chromatin. Genome Res. 20, 899-907 (2010).

29. Kaneko, S. et al. Interactions between JARID2 and noncoding RNAs regulate PRC2 recruitment to chromatin. Mol. Cell 53, 290-300 (2014).

30. Modarresi, F. et al. Inhibition of natural antisense transcripts in vivo results in gene-specific transcriptional upregulation. Nat. Biotechnol. 30, 453-459 (2012). 
31. Zhang, X. et al. Maternally expressed gene 3 (MEG3) noncoding ribonucleic acid: isoform structure, expression, and functions. Endocrinology 151, 939-947 (2010).

32. Wiercinska, E. et al. The TGF-beta/Smad pathway induces breast cancer cell invasion through the up-regulation of matrix metalloproteinase 2 and 9 in a spheroid invasion model system. Breast Cancer Res. Treat. 128, 657-666 (2011).

33. Padua, D. et al. TGFbeta primes breast tumors for lung metastasis seeding through angiopoietin-like 4. Cell 133, 66-77 (2008).

34. Buijs, J. T., Stayrook, K. R. \& Guise, T. A. The role of TGF-beta in bone metastasis: novel therapeutic perspectives. Bonekey Rep. 1, 96 (2012).

35. Mariner, P. D. et al. Human Alu RNA is a modular transacting repressor of mRNA transcription during heat shock. Mol. Cell 29, 499-509 (2008).

36. Simon, M. D. et al. The genomic binding sites of a noncoding RNA. Proc. Nat Acad. Sci. USA 108, 20497-20502 (2011).

37. McLean, C. Y. et al. GREAT improves functional interpretation of cisregulatory regions. Nat. Biotechnol. 28, 495-501 (2010).

38. Martianov, I., Ramadass, A., Serra Barros, A., Chow, N. \& Akoulitchev, A. Repression of the human dihydrofolate reductase gene by a non-coding interfering transcript. Nature 445, 666-670 (2007).

39. Khomyakova, E. B. et al. Parallel intramolecular DNA triple helix with G and T bases in the third strand stabilized by $\mathrm{Zn}(2+)$ ions. Nucleic Acids Res. 28, 3511-3516 (2000).

40. Besch, R., Giovannangeli, C., Kammerbauer, C. \& Degitz, K. Specific inhibition of ICAM-1 expression mediated by gene targeting with Triplex-forming oligonucleotides. J. Biol. Chem. 277, 32473-32479 (2002).

41. Buske, F. A., Bauer, D. C., Mattick, J. S. \& Bailey, T. L. Triplexator: Detecting nucleic acid triple helices in genomic and transcriptomic data. Genome Res. 22, 1372-1381 (2012)

42. Xodo, L. E., Manzini, G. \& Quadrifoglio, F. Spectroscopic and calorimetric investigation on the DNA triplex formed by d(CTCTTCTTTCTTTTCTTTCTTCTC) and d(GAGAAGAAAGA) at acidic pH. Nucleic Acids Res. 18, 3557-3564 (1990).

43. Manzini, G. et al. Triple helix formation by oligopurine-oligopyrimidine DNA fragments. Electrophoretic and thermodynamic behavior. J. Mol. Biol. 213, 833-843 (1990)

44. Scaria, P. V., Will, S., Levenson, C. \& Shafer, R. H. Physicochemical studies of the d(G3T4G3)*d(G3A4G3).d(C3T4C3) triple helix. J. Biol. Chem. 270, 7295-7303 (1995).

45. Gorab, E., Amabis, J. M., Stocker, A. J., Drummond, L. \& Stollar, B. D. Potential sites of triple-helical nucleic acid formation in chromosomes of Rhynchosciara (Diptera: Sciaridae) and Drosophila melanogaster. Chromosome Res. 17, 821-832 (2009)

46. Stollar, B. D. \& Raso, V. Antibodies recognise specific structures of triple-helical polynucleotides built on poly(A) or poly(dA). Nature 250, 231-234 (1974).

47. Fadloun, A. et al. Chromatin signatures and retrotransposon profiling in mouse embryos reveal regulation of LINE-1 by RNA. Nat. Struct. Mol. Biol. 20, 332-338 (2013)

48. Ohno, M., Fukagawa, T., Lee, J. S. \& Ikemura, T. Triplex-forming DNAs in the human interphase nucleus visualized in situ by polypurine/polypyrimidine DNA probes and antitriplex antibodies. Chromosoma 111, 201-213 (2002)

49. Annex, B. H. \& Williams, R. S. Mitochondrial DNA structure and expression in specialized subtypes of mammalian striated muscle. Mol. Cell. Biol. 10, 5671-5678 (1990).

50. Doda, J. N., Wright, C. T. \& Clayton, D. A. Elongation of displacement-loop strands in human and mouse mitochondrial DNA is arrested near specific template sequences. Proc. Natl Acad. Sci. USA 78, 6116-6120 (1981).

51. Rinn, J. L. et al. Functional demarcation of active and silent chromatin domains in human HOX loci by noncoding RNAs. Cell 129, 1311-1323 (2007).

52. Han, L. et al. Low expression of long noncoding RNA GAS6-AS1 predicts a poor prognosis in patients with NSCLC. Med. Oncol. 30, 694 (2013).

53. Footz, T. K. et al. Analysis of the cat eye syndrome critical region in humans and the region of conserved synteny in mice: a search for candidate genes at or near the human chromosome 22 pericentromere. Genome Res. 11, 1053-1070 (2001).

54. Chevli, K. K. et al. Urinary PCA3 as a predictor of prostate cancer in a cohort of 3,073 men undergoing initial prostate biopsy. J. Urol. 191, 1743-1748 (2013).

55. Xu, K. et al. EZH2 oncogenic activity in castration-resistant prostate cancer cells is polycomb-independent. Science 338, 1465-1469 (2012).

56. Lee, S. T. et al. Context-specific regulation of NF-kappaB target gene expression by EZH2 in breast cancers. Mol. Cell 43, 798-810 (2011).

57. Mondal, T. \& Kanduri, C. Maintenance of epigenetic information: a noncoding RNA perspective. Chromosome Res. 21, 615-625 (2013).

58. Mohammad, F. et al. Long noncoding RNA-mediated maintenance of DNA methylation and transcriptional gene silencing. Development 139, 2792-2803 (2012)
59. He, Y. et al. Inhibitory effects of long noncoding RNA MEG3 on hepatic stellate cells activation and liver fibrogenesis. Biochim. Biophys. Acta 1842, 2204-2215 (2014).

60. Schmitz, K. M., Mayer, C., Postepska, A. \& Grummt, I. Interaction of noncoding RNA with the rDNA promoter mediates recruitment of DNMT3b and silencing of rRNA genes. Genes Dev. 24, 2264-2269 (2010).

61. Beckedorff, F. C. et al. The intronic long noncoding RNA ANRASSF1 recruits PRC2 to the RASSF1A promoter, reducing the expression of RASSF1A and increasing cell proliferation. PLoS Genet. 9, e1003705 (2013)

62. Wang, X. et al. Induced ncRNAs allosterically modify RNA-binding proteins in cis to inhibit transcription. Nature 454, 126-130 (2008).

63. Bertani, S., Sauer, S., Bolotin, E. \& Sauer, F. The noncoding RNA Mistral activates Hoxa6 and Hoxa7 expression and stem cell differentiation by recruiting MLL1 to chromatin. Mol. Cell 43, 1040-1046 (2011).

64. Cifuentes-Rojas, C., Hernandez, A. J., Sarma, K. \& Lee, J. T. Regulatory interactions between RNA and polycomb repressive complex 2. Mol. Cell 55, 171-185 (2014).

65. Horard, B., Tatout, C., Poux, S. \& Pirrotta, V. Structure of a polycomb response element and in vitro binding of polycomb group complexes containing GAGA factor. Mol. Cell Biol. 20, 3187-3197 (2000).

66. Klymenko, T. et al. A Polycomb group protein complex with sequence-specific DNA-binding and selective methyl-lysine-binding activities. Genes Dev. 20, 1110-1122 (2006).

67. Deng, W. et al. Arabidopsis polycomb repressive complex 2 binding sites contain putative GAGA factor binding motifs within coding regions of genes. BMC Genomics 14, 593 (2013).

68. Woo, C. J., Kharchenko, P. V., Daheron, L., Park, P. J. \& Kingston, R. E. A region of the human $\mathrm{HOXD}$ cluster that confers polycomb-group responsiveness. Cell 140, 99-110 (2010).

69. Sing, A. et al. A vertebrate Polycomb response element governs segmentation of the posterior hindbrain. Cell 138, 885-897 (2009)

70. Kuo, M. H. \& Allis, C.D. In vivo cross-linking and immunoprecipitation for studying dynamic Protein:DNA associations in a chromatin environment. Methods 19, 425-433 (1999).

\section{Acknowledgements}

We acknowledge the Centre for Cellular Imaging at the Sahlgrenska Academy, University of Gothenburg, for the image acquisition and analysis, and Array and Analysis Facility, Science for Life Laboratory at Uppsala Biomedical Center, Husargatan 3, 75123 Uppsala. This work was supported by the grants from the Knut and Alice Wallenberg Foundation (Dnr KAW 2014.0057), Swedish Foundation for Strategic Research (RB13-0204), Swedish Cancer Research foundation (Cancerfonden: Kontrakt no. 140317), the Swedish Research Council (VR-M: K2014-67X-20781-07-4), Barncancerfonden (PR2014/0147), Biocare/GU and LUA/ALF to C.K. The work of C.M.G. is supported by the Swedish Cancer Foundation (Cancerfonden) and the Swedish Research Council (VR). The work of F.W. is supported by the Area of Advance in Nanoscience and Nanotechnology at Chalmers University of Technology.

\section{Author contributions}

T.M and C.K designed the research and prepared the manuscript. T.M, R.V, S.U, B.R, S.M, A.M, E.H, F.W and E.G performed the experiments. S.S, S.E, A.R.J and A.H.S analysed the data. A.M, U.G, S.J and C.M.G provided valuable support.

\section{Additional information}

Accession codes: The data associated with this publication have been deposited in European Nucleotide Archive and are accessible through accession number PRJEB7307.

Supplementary Information accompanies this paper at http://www.nature.com/ naturecommunications

Competing financial interests: The authors declare no competing financial interests

Reprints and permission information is available online at http://npg.nature.com/ reprintsandpermissions/

How to cite this article: Mondal, T. et al. MEG3 long noncoding RNA regulates TGF- $\beta$ pathway genes through formation of RNA-DNA triplex structures. Nat. Commun 6:7743 doi: 10.1038/ncomms8743 (2015).

This work is licensed under a Creative Commons Attribution 4.0 International License. The images or other third party material in this article are included in the article's Creative Commons license, unless indicated otherwise in the credit line; if the material is not included under the Creative Commons license, users will need to obtain permission from the license holder to reproduce the material. To view a copy of this license, visit http://creativecommons.org/licenses/by/4.0/ 